\title{
Envelope-Domain Analysis and Modeling of Super-Regenerative Oscillators
}

\author{
Silvia Hernández, Student Member, IEEE, Almudena Suárez, Fellow, IEEE
}

\begin{abstract}
An envelope-domain methodology for the numerical modeling of super-regenerative oscillators (SRO) is presented. The main advantage is its generality of application to transistor-based oscillators with arbitrary topology. Initially, a stability analysis of the non-oscillatory steady-state solution, forced by the quench signal, is performed. It is based on the calculation of a linear timevariant (LTV) transfer function, obtained by linearizing the circuit envelope-domain equations about the non-oscillatory regime. Under moderate quench frequencies, it will be possible to estimate the SRO normalized envelope and sensitivity function from the detected dominant pair of complex-conjugate poles. In the general case, the SRO oscillatory response is modeled with a numerical method, valid under linear operation with respect to the input signal. This is based on the calculation of the LTV impulse response from a time-frequency transfer function obtained under a small-signal sinusoidal excitation. The LTV impulse response enables a straightforward determination of the sensitivity time interval and time distance to the envelope maximum. An integral expression, in terms of the LTV transfer function, will provide the SRO response to any small signal input, with any arbitrarily carrier frequency and modulation. The methodology has been successfully validated through its application to a $\mathrm{SRO}$ at $2.7 \mathrm{GHz}$, which has been manufactured and measured.
\end{abstract}

Index Terms - Linear-time-variant transfer function, superregenerative oscillator, stability.

\section{INTRODUCTION}

$\mathrm{S}$ UPER-REGENERATIVE OSCILLATORS (SROs), which are periodically switched on and off by a quench signal [1][5], enable a high gain amplification of a small input signal near the oscillator resonant frequency. This amplification is due to the fast amplitude growth during each oscillation start-up cycle [1]-[5], which is exponential in its initial stage. SROs have recently attracted significant attention since they can replace chains of several lower gain amplifiers, enabling a reduction of power consumption, which has a special interest at millimeter and Terahertz frequencies [6]-[7]. In some recent works [6]-[9], they have been applied for the implementation of compact receivers, with amplitude, frequency and phase demodulation capabilities. The SRO concept has also been extended to the implementation of low consumption active transponders [10]-[11], where the oscillator-based transponder provides a high-amplitude response to the interrogating signal. The high-gain amplification associated with the superregenerative effect allows for compact transponders with a long-range operation.

This work was supported by the Spanish Ministry of Economy and Competitiveness under the research project TEC2014-60283-C3-1-R. S. Hernández and A. Suárez are with the Departamento de Ingeniería de
The works [2], [4]-[5] present a thorough and insightful formulation for the super-regenerative receiver, based on the approximate analytical resolution of the second-order differential equation system that describes the switched oscillator in the presence of a weak input signal. A closed-form expression of the solution [2] is derived, in terms of superregenerative and regenerative gains, sensitivity function and normalized envelope. These functions, which enable an indepth understanding of the SRO operation, have been fundamental for many subsequent works, exploring novel methodologies for SRO design and optimization [4]-[8], [12]. However, due to certain assumptions on the magnitude and time derivative of the quench signal, the analytical solution has some validity limitations and is usable for relatively low quench frequencies, in a certain quality-factor range. To predict the oscillator instantaneous frequency and phase, alternative ways such as [3] or [13] may be used.

Another problem comes from the fact that the SRO is modeled in a simplified manner, by means of a resonator with a single voltage-controlled current source as an active element, or a single-loop feedback system, made up of a passive and an active block, with explicit transfer characteristics. These simplified models might not accurately represent transistorbased oscillators, in which the models of the various components or blocks are not easily identified. For a more realistic prediction of the oscillator response, numerical methods, such as time-domain integration or envelope transient are needed. In fact, envelope transient [14]-[20] should be the most efficient method for the SRO analysis due to the presence of widely separated time scales, corresponding to the quench and modulation signals, on the one hand, and the oscillation and input carrier, on the other hand. However, the envelope transient simulation of SROs has been mostly limited to the usual simplified models. For instance, in [3], envelope transient is applied for the validation of an interesting frequency-domain analysis of a super-regenerative receiver, represented as a feedback system. The cross-coupled transistors, which constitute the active part of the switched oscillator, are modeled with a cubic nonlinearity and a hyperbolic-tangent gain function. In [21] a baseband/lowpass equivalent model of the SRO based on [2] is derived, which significantly reduces the computational cost. In addition, the works [13], [22] present more precise and efficient computation methods of the SRO response (envelope and phase) than the classical approach.

Comunicaciones, Universidad de Cantabria, Santander, 39005, Spain (e-mail: silvia.hernandez@unican.es; almudena.suarez@unican.es). 
The aim of this work is to provide a numerical analysis methodology, applicable to any SRO behaving in linear mode, regardless of the particular topology of the oscillator circuit, most often based on transistor devices. The method relies on the definition of an envelope-domain oscillatory LTV impulse response, which accurately characterizes the SRO response, in terms of the sensitivity time interval [2] and time value with maximum gain, and an oscillatory LTV transfer function. The functions are easily extracted through envelope-transient simulations at circuit level, under simple small-signal sinusoidal excitations. These functions enable an efficient prediction of the SRO response under arbitrarily modulated inputs signals, which is done in in-house software, without having to perform any circuit-level simulations. This way a compact behavioral model of the SRO is derived, usable on system-level simulations.

The methodology will be based on the envelope-transient technique [14]-[20], in combination with Zadeh's frequencyanalysis of time-varying networks [23]-[25]. The first stage of this methodology consists of the identification of the timevarying poles associated with the non-oscillatory homogeneous solution of the SRO, in which the circuit simply responds to the quench signal, without any oscillation. This is done by defining a LTV transfer function [23] fulfilling all the properties established in [23]-[25]. The time varying poles will enable the determination of the time references in the SRO operation, e.g., the time values at which the dominant complex-conjugate poles cross the imaginary axis (in the two senses) during the oscillation period. In a second stage, a procedure is presented to obtain the envelope-domain oscillatory LTV impulse response that globally characterizes the SRO behavior under a given quench signal, which is valid in the commonly used linearoperation mode. This impulse response, which is not subject to any approximations, gives a useful insight into the circuit behavior, as it enables an easy identification of the sensitivity time interval and time distance to the envelope maximum. Then, an integral expression, in terms of the oscillatory LTV transfer function, will provide the SRO response to any smallsignal input, with arbitrary carrier frequency and modulation.

The methodology is of general application to any SRO topology. As stated, the new LTV functions are extracted through a circuit-level envelope-transient simulation of the oscillator, excited with a small-signal sinusoidal source. This is used for either the stability analysis of the non-oscillatory regime forced by the quench signal, or for the SRO envelopedomain characterization in oscillatory regime. There is no limiting assumption preventing the generality of the analysis methodology.

All the results are validated through comparison with circuitlevel envelope-transient analyses. This ensures identical circuitcomponent models in the envelope-transient simulations and in the specific simulations performed to extract the LTV impulse response and transfer function. The methodology has also been experimentally validated through its application to a SRO at 2.7 $\mathrm{GHz}$, which has been manufactured and measured.

The paper is organized as follows. Section II presents the general envelope-domain formulation. Section III describes the stability analysis of the non-oscillatory solution. Section IV presents the envelope-domain oscillatory LTV impulse response. Section V describes the calculation to of the SRO output signal under arbitrarily modulated inputs. Section VI describes the experimental measurements.

\section{ENVELOPE-TRANSIENT ANALYSIS}

The modified-nodal analysis (MNA) of a given nonlinear circuit is based on the application to this circuit of Kirchoff's laws [17], [26] taking into account the constitutive relationships of its elements or components. This provides the following general system of differential equations, in vector form [27][30]:

$\bar{f}(\bar{x}(t))+\frac{d \bar{q}(\bar{x}(t))}{d t}+\int_{-\infty}^{t}[d(t-\tau)] \bar{x}(\tau) d \tau+\bar{g}(t)=0$

where $\bar{x}$ is the vector containing the node voltages and branch currents that cannot be expressed in terms of node voltages and/or their first order derivatives [30], $\bar{f}$ is the vector of containing the linear and nonlinear resistive terms, $\bar{q}$ is the vector of linear and nonlinear charges and fluxes, $[d]$ is a matrix containing the impulsive responses of the distributed elements, and $\bar{g}(t)$ are the input generators.

In circuits containing two different time scales, system (1) can be efficiently solved using the envelope-transient method [14]-[20]. This is the case of SROs, schematically represented in Fig. 1. In this circuit, the oscillation is switched on and off by a quench-signal $V_{q}(t)$ at the frequency $\omega_{q}$, which is much lower than the oscillation frequency $\omega_{o}$. The fast amplitude growth during each oscillation start-up cycle enables a highgain amplification of a small input signal at the carrier frequency $\omega$. The input frequency $\omega$ must be relatively close to $\omega_{o}$ to achieve amplification. As shown in Fig. 1, two different time scales can be distinguished: a slow time scale, corresponding to the quench signal, input modulations and frequency difference $\left|\omega-\omega_{o}\right|$, and a fast time scale corresponding to the high frequency $\omega$.

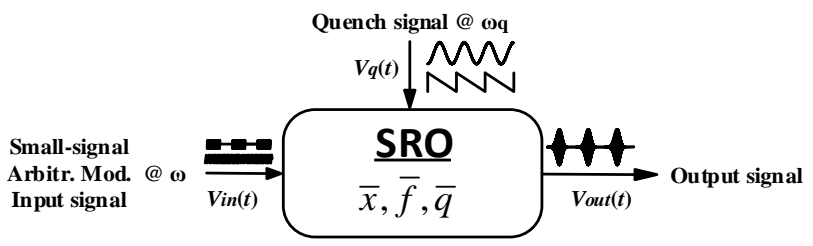

Fig. 1. General representation of the SRO indicating the different signals and time scales.

In the envelope-transient method, the circuit variables [including $\bar{x}(t), \bar{f}(t), \bar{q}(t)$ and $\bar{g}(t)]$ are represented in a Fourier series with time varying coefficients, which can have an arbitrary time variation. Assuming a representative variable $y(t)$, the envelope-domain representation is as follows:

$y(t)=\sum_{k=-N H}^{N H} Y_{k}(t) e^{j k \omega t}$

where $\omega$ is the high fundamental frequency and $Y_{k}(t)$ are the time-varying harmonic coefficients. In the remainder of the 
manuscript, low case will be used for full time-domain state variables and capitals will be used for time-varying Fourier coefficients. These coefficients (or complex envelopes) must vary slowly in comparison with $\omega$ since for a coefficient bandwidth larger than $\omega / 2$ the envelopes will overlap, and the system solution will not be unique [17]. This requirement is analogous to the bandwidth constraint in the lowpass equivalent of bandpass signals [31]. Under this situation, the only limitation of (2) would be associated with the possible existence of a frequency component that is not considered in this representation. This can be an additional high-frequency oscillation that is neither taken into account in the frequency basis nor in the envelope time variation. Nevertheless, the possible presence of additional oscillation frequencies should be detectable through a complementary stability analysis [32][36].

To derive the envelope-transient formulation, the variable representation (2) is introduced in the MNA of (1). It is taken into account that the derivatives of the reactive elements $\bar{q}$ are expressed:

$\dot{q}_{m}(t)=\sum_{k=-N H}^{N H}\left(\dot{Q}_{m, k}(t)+j k \omega\right) e^{j k \omega t}$

where $m$ refers to a particular reactive element. After substitution into (1) and elimination of the exponentials $e^{j k \omega t}$, one obtains the following general expression of the envelopetransient system [14]-[20]:

$\bar{F}(\bar{X}(t))+[j \omega] \bar{Q}(\bar{X}(t))+\frac{d \bar{Q}(\bar{X}(t))}{d t}+$

$[D(t)] * \bar{X}(t)+\bar{G}(t)=0$

where $\bar{F}$ is the vector containing the time-varying harmonic components (at $k \omega$ ) of the resistive elements, $\bar{Q}$ is an analogous vector, corresponding to the reactive elements, $[j \omega]$ is the matrix accounting for the derivatives of the complex exponential terms in $(2),[D(t)]$ is the matrix containing the time-varying harmonic components of the impulse responses, associated to the distributed elements, and $\bar{G}$ is the vector of input sources. System (4) is a differential equation system in the slowly-varying Fourier components of the circuit state variables, which can be integrated at a much larger time step than the ordinary transient analysis. It is thus more efficient. Its practical resolution, after discretizing the time variable, through an error-minimization algorithm, is explained in detail in [17], [19], [27]-[29].

In the SRO analysis, the fundamental frequency $\omega$ of the envelope-domain representation (2) will be the carrier frequency of the existing high-frequency independent source. In ordinary operation of the SRO, the vector $\bar{G}(t)$ will contain the quench signal $V_{q}(t)$, as a baseband component, and the lowpass equivalent of the bandpass input signal $v_{i n}(t)$, at the carrier frequency $\omega$. It can be written as:

$\bar{G}(t)=\left[\begin{array}{lllllll}0 & \cdots & \tilde{V}_{i n}{ }^{*}(t) & V_{q}(t) & \tilde{V}_{i n}(t) & \cdots & 0\end{array}\right]^{T}$

When applied to the SRO, system (4) is homogeneous with respect to the oscillation frequency, so it will exhibit two different solutions: an oscillatory solution, $\bar{X}_{o}(t)$, switched on and off by the quench signal, and a non-oscillatory one, $\bar{X}_{q}(t)$. The low-frequency solution $\bar{X}_{q}(t)$, without any oscillation, results from the forcing by the quench signal $V_{q}(t)$ at the frequency $\omega_{q}$. It is conceptually analogous to the dc solution for which any free-running oscillator can be solved. The signal $V_{q}(t)$ constitutes a baseband term, so $\bar{X}_{q}(t)$ will be at baseband too. To enable the oscillation start-up, $\bar{X}_{q}(t)$ should be unstable during a time interval of the quench-signal period $T_{q}$. On the other hand, the oscillatory solution $\bar{X}_{o}(t)$ will contain high frequency components. The number of harmonic components in (2) will be $N H=1$, in the linear mode, and $N H>1$, in the logarithmic mode, due to the generation of harmonic content by the oscillation signal [1].

TABLE I

DIFFERENCES BETWEEN THE NEW ANALYSIS PROCEDURE AND THE CONVENTIONAL ENVELOPE-TRANSIENT

Method Characteristics

Circuit-level - Individual envelope-transient simulation for envelope each particular (modulated) input signal.

transient - No information about the frozen or non-frozen behavior of the SRO.

- No direct information on the sensitivity interval.

- Simulation time of an impulse response in Fig. 9(b): $1.85 \mathrm{~s}$

\begin{tabular}{ll}
\hline $\begin{array}{l}\text { Envelope- } \\
\text { domain LTV }\end{array}$ & - The LTV functions are easily calculated \\
impulse & through circuit-level envelope transient \\
response and & simulation under sinusoidal excitations only. \\
transfer function & - Information on SRO frozen or non-frozen \\
& behavior from time-varying poles of LTV \\
& transfer function $H_{s}(t, \omega)$ \\
& - Full characterization of sensitivity interval \\
& and gain response through the evaluation of the \\
& oscillatory LTV impulse response $\tilde{h}(t, \tau)$, as \\
& shown in the algorithm of Table II. \\
& - Calculation of response to arbitrarily \\
& modulated input signals using the extracted \\
& oscillatory LTV transfer function $H(t, \omega)$, as \\
& shown in the algorithm of Table III. \\
& - Simulation time of an impulse response in Fig. \\
& $9(b): 0.65 \mathrm{~s}$
\end{tabular}

Without any small high-frequency perturbation, system (4) converges towards $\bar{X}_{q}(t)$. This situation is conceptually identical to that in the time-domain analysis of free-running oscillators, which requires a small perturbation of the unstable dc solution to initiate the transient to the oscillatory regime.

However, in the presence of a high-frequency input or perturbation, system (4) may converge to either the oscillatory or non-oscillatory solution. This depends on the integration time step. In the non-oscillatory solution, the time-varying phasors are sampled at the rate of the quench signal. In the oscillatory solution, they are sampled at the rate of envelope of the oscillation pulses. Obtaining the oscillatory solution 
requires a small integration time step (though still much larger than in a standard time-domain integration), so that the envelope-domain system may be able to capture the oscillation dynamics. This integration should account for the difference between $\omega$ and the oscillation frequency $\omega_{o}$, expressed as $\left|\omega-\omega_{o}\right|$, and be able to provide the envelope of the oscillation pulse.

The new investigation provides an analysis methodology of SROs in two stages. The first stage is a stability analysis of the non-oscillatory solution $\bar{X}_{q}(t)$. The second stage is the calculation of the SRO envelope-domain oscillatory LTV impulse response and oscillatory LTV transfer function, which fully characterize the SRO response in linear mode. Differences between the new analysis procedure and the conventional envelope-transient method are summarized in Table I.

\section{LTV TRANSFER FUNCTION OF THE NON-OSCILLATORY SOLUTION}

As stated in the previous section, in the absence of perturbations, system (4) admits a solution $\bar{X}_{q}(t)$ at the frequency $\omega_{q}$ of the quench signal, without any oscillation. This section presents the stability analysis of $\bar{X}_{q}(t)$ and shows how the results of this analysis can be used to identify the SRO functions defined in [2], more specifically, the normalized envelope of the homogeneous solution $p(t)$, the sensitivity function $s(t)$ and the super-regenerative gain $K_{s}$.

\section{A. Foundations of the stability analysis}

The stability of the solution $\bar{X}_{q}(t)$ is analyzed by introducing into the circuit a small-signal sinusoidal current source $i_{p}(t)$ at the frequency $\omega[29]-[30]$. In the presence of this small-signal source, the vector of state variables is expressed as [31]:

$\bar{x}(t)=\bar{X}_{q}(t)+\Delta \bar{X}_{1}(t) e^{j \omega t}+\Delta \bar{X}_{-1}(t) e^{-j \omega t}$

The system response to the small-signal source $i_{p}(t)$ can be characterized by linearizing (4) with respect to this source and obtaining an LTV impulse response $h(t, \tau)$ [23]-[25], where $t$ corresponds to the quench-signal rate and $\tau$ indicates the instant when the impulse is applied. The output signal, in terms of a particular state variable $x_{m}(t)$, corresponding, for instance, to a node voltage, can be expressed as:

$x_{m}(t)=\int_{-\infty}^{\infty} h_{s}(t, \tau) i_{p}(\tau) d \tau$

where the subindex $s$ is used to refer to functions associated to the non-oscillatory solution. Then, a slowly-varying transfer function at the slow time rate $t$ can be obtained doing [23]:

$H_{s}(t, \omega)=\int_{-\infty}^{\infty} h_{s}(t, \tau) e^{-j \omega(t-\tau)} d \tau$

The above function can also be written in terms of the complex frequency $s$ [23]-[25], with a formally identical expression: $H_{s}(t, s)$. The LTV network will be stable if the function $H_{s}(t, s)$ is analytic and bounded in the right half side of the complex plane (RHS) and in the imaginary axis for all time $t$ [25]. In fact, as stated in [25], the network will be stable if the poles of $H_{s}(t, s)$, whose location varies with time, do not cross to the right-hand side of the complex plane (RHS). The time- varying poles of systems with two different time scales have also been formally derived in [39], where the analysis departs from a system of multirate partial differential equations. In [32], time-varying poles of circuits driven with modulated inputs have been calculated through identification procedures.

As shown in [23]-[25], the LTV transfer function $H_{s}(t, s)$ of the linearized-system can be obtained by introducing an input signal $e^{j \omega t}$ and calculating the ratio between the resulting output $x_{m, \omega}(t)$ at the frequency $\omega$ and the input $e^{j \omega t}$ :

$H_{s}(t, \omega)=\frac{x_{m, \omega}(t)}{e^{j \omega t}}$

To determine the transfer function (9), expression (6) is introduced in (2). Because $\Delta \bar{X}_{1}(t), \Delta \bar{X}_{-1}(t)$ are small, it is possible to perform a first-order Taylor series expansion of the two vectors $\bar{F}(\bar{X}(t)), \bar{Q}(\bar{X}(t))$ in (2) about the nonoscillatory solution $\bar{X}_{q}(t)$. This Taylor-series expansion provides the following linear differential-equation system:

$$
\begin{aligned}
& \frac{d}{d t}\left[\frac{\partial \bar{Q}_{0}\left(\bar{X}_{q}(t)\right)}{\partial \bar{X}_{0}} \Delta \bar{X}_{1}(t)\right]= \\
& -\left\{\frac{\partial \bar{F}_{0}\left(\bar{X}_{q}(t)\right)}{\partial \bar{X}_{0}}+[j \omega] \frac{\partial \bar{Q}_{0}\left(\bar{X}_{q}(t)\right)}{\partial \bar{X}_{0}}\right\} \Delta \bar{X}_{1}(t)-\bar{I}_{p}=0
\end{aligned}
$$

where the Jacobian matrixes contain the derivatives of the baseband reactive elements and resistive elements and $\bar{I}_{p}$ is a vector containing $I_{p}$. Taking (9) into account, the time-variant transfer function, associated to the $m^{\text {th }}$ state variable, $H_{s, m}(t, \omega)$ , is calculated as:

$$
H_{s, m}(t, \omega)=\frac{\Delta X_{1, m}(t)}{I_{p}} \equiv \frac{x_{m, \omega}(t)}{e^{j \omega t}}
$$

where $I_{p}$ is the magnitude of the small-signal current source and the sub-indexes $1, m$ indicate the component at the positive frequency $\omega$ of the $m^{\text {th }}$ state variable. For a better insight, system (2) will be particularized to a circuit without distributed elements, though all results are equally applicable to a circuit containing these elements. Because $\Delta X_{1, m}(t)$ in (11) is a solution of (10), any possible transfer function will fulfill the linear differential equation system with time-varying coefficients in (10). This is in full agreement with Zadeh's theory [23]-[25].

\section{B. Frozen and non-frozen behavior}

Several cases can be distinguished [23]-[25]: a "frozen system", for which the time derivatives of the envelopes are negligible $\Delta \dot{\bar{X}}(t)=0$, an "intermediate" system that is nonfrozen but exhibits variations at a time rate much lower than the carrier frequency $\omega$ and a faster system, at higher time rate, but still enabling the variable representation (6). If the system is frozen, its stability properties will be identical to those obtained by eliminating the time derivative in (10) and replacing the time variable in the quench signal with an artificial parameter $\eta$, which should be swept in the same range as the original time variable. 
In an intermediate system, it should be possible to apply a perturbation approach similar to the one in [24], which would allow the calculation of the transfer function as a series:

$$
H_{s, m}(t, \omega) \cong H_{s, m, 1}(t, \omega)+H_{s, m, 2}(t, \omega)+\ldots
$$

where the first function $H_{s, m, 1}(t, \omega)$ corresponds to the solution of the frozen system, given by:

$$
\Delta \bar{X}_{1,1}(t)=\left\{\frac{\partial \bar{F}_{0}\left(\bar{X}_{q}(t)\right)}{\partial \bar{X}_{0}}+[j \omega] \frac{\partial \bar{Q}_{0}\left(\bar{X}_{q}(t)\right)}{\partial \bar{X}_{0}}\right\}^{-1} \bar{I}_{p}
$$

And the rest of terms are calculated in the following recursive form:

$$
\begin{aligned}
& \Delta \bar{X}_{1, n}(t)= \\
& \left\{\frac{\partial \bar{F}_{0}\left(\bar{X}_{q}(t)\right)}{\partial \bar{X}_{0}}+[j \omega] \frac{\partial \bar{Q}_{0}\left(\bar{X}_{q}(t)\right)}{\partial \bar{X}_{0}}\right\}^{-1} \frac{d}{d t}\left[\frac{\partial \bar{Q}_{0}\left(\bar{X}_{q}(t)\right)}{\partial \bar{X}_{0}} \Delta \bar{X}_{n-1}(t)\right]
\end{aligned}
$$

Note that systems (10) and (12)-(14) are two different ways of computing the same result, though the expansion (12)-(14) is an approximation of (10). In fact, as the frequency of the quench signal increases, the accuracy of the approach (12) degrades and becomes inapplicable, due to the high value of the time derivative in (10). Other effects, including the circuit stabilization, may arise. Stabilization as the frequency of the quench signal increases is a natural phenomenon since when entering and exiting the unstable region very quickly due to a high-frequency quench signal, the oscillation will not have enough time to start up.

\section{Application example}

The analysis in (10) and (12)-(14) will be illustrated through its application to the simple circuit shown in Fig. 2. It contains a single resonator and a negative-resistance element. The quality factor of the RLC resonator is about $Q_{o}=12$. To compare with [2], the quench signal will be directly introduced in the cubic nonlinearity that describes the active device. Note that this direct introduction is not possible in practice. The active device will be modeled with the following function:

$i[v(t), a(t)]=a(t) v(t)+b v^{3}(t)$

In the linear mode, the coefficient $b$ can be neglected. The time-varying coefficient $a(t)$ is given by:

$$
a(t)=A_{d c}+A_{p} \cos \left(\omega_{q} t\right)
$$

where $A_{d c}$ is defined as a positive quantity, $A_{p}$ is the peak amplitude of the time-varying quench signal and $\omega_{q}$ is the frequency of this signal. Introducing a small signal current source $i_{p}(t)$, at the frequency $\omega$, in the circuit of Fig. 2, system (10) particularizes to:

$$
\begin{aligned}
& \dot{I}_{L, 1}(t)=\frac{V_{c, 1}(t)}{L}-j \omega I_{L, 1}(t) \\
& \dot{V}_{c, 1}(t)=-\frac{1}{C}\left(a(t) V_{c, 1}(t)+I_{L, 1}(t)+\frac{(1+j \omega R C)}{R} V_{c, 1}(t)\right)+\frac{I_{p}}{C}
\end{aligned}
$$

The transfer function considered is the following:

$$
Z(t, \omega)=\frac{V_{c, 1}(t, \omega)}{I_{p}}
$$

The effect of $\omega_{q}$ on $Z(t, \omega)$ will be analyzed comparing the results obtained through the integration of (17), which is a particularization of (10) to the circuit in Fig. 2, and through the perturbation method in (12)-(14). This will illustrate the system deviation from the "frozen" conditions when increasing $\omega_{q}$.

Fig. 3 presents the waveforms of the $Z(t, \omega)$ magnitude obtained for the same excitation frequency $\omega=2 \pi 2.56 \mathrm{e} 9 \mathrm{rad} / \mathrm{s}$ and two different values of the quench frequency. Results obtained with (17) are compared with those obtained with the approximation (12)-(14). For $f_{q}=1 \mathrm{kHz}$ [Fig. 3(a)], the system behaves as frozen. In a frozen situation, the time-derivatives in (17) are so small that they have no impact on the circuit behavior. Thus, the function (18) obtained by using the voltage $V_{c, 1}(t, \omega)$ from the integration of (17) agrees with the function (18) obtained by solving (17) for $V_{c, 1}(t, \omega)$ under the conditions $\dot{I}_{L, 1}(t)=\dot{V}_{c, 1}(t)=0$.

For $f_{q}=1 \mathrm{MHz}$ [Fig. 3(b)], the system is non-frozen, since the function $Z(t, \omega)$ is different from the one obtained neglecting the time derivatives, shown in Fig. 3(a). In Fig. 3(b), the function $Z(t, \omega)$ obtained after one iteration of the perturbation method in (12)-(14) is nearly overlapped with the one obtained through the integration of (17) (which is a particularization of (10) to the circuit in Fig. 2) .

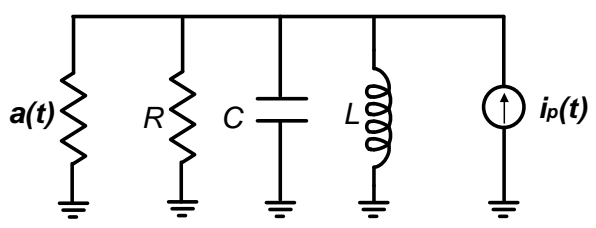

Fig. 2. Oscillator based on a negative resistor. Element values are $C=15.0642$ $\mathrm{pF}, L=0.2473 \mathrm{nH}, R=50 \Omega, a(t)=A_{d c}+A_{p} \cos \left(\omega_{q} t\right)$ where $A_{d c}=0.01 \Omega^{-1}$ and $A_{p}=0.0337 \Omega^{-1}$. The quality factor of the RLC resonator is about $Q_{o}=12$.
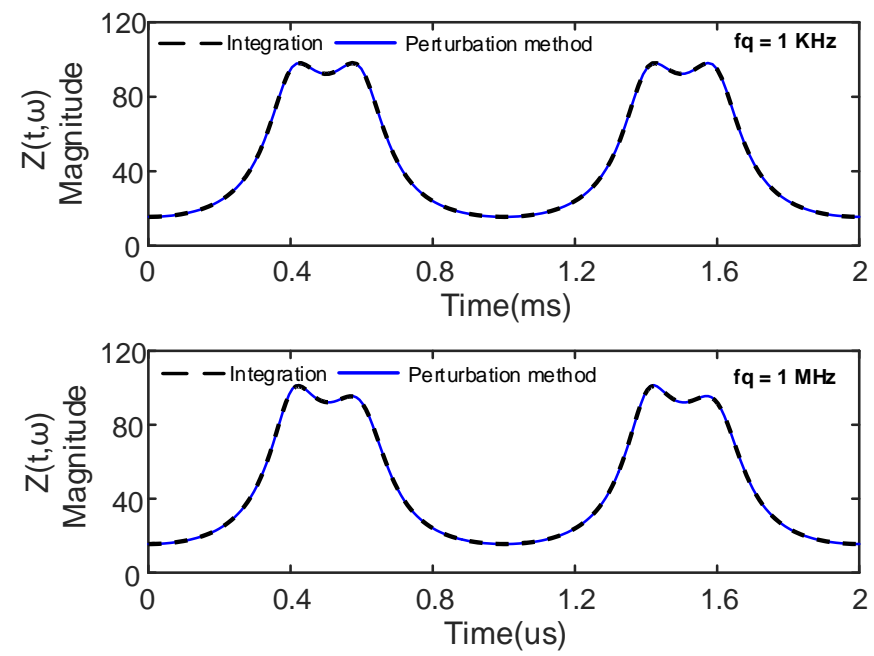

Fig. 3. Comparison between the waveforms of the $Z(t, \omega)$ magnitude obtained through integration of (17) and through the perturbation method in (12)-(14) for different values of the quench frequency $\omega_{q}$. The waveforms have been calculated for the excitation frequency $\omega=2 \pi 2.56 \mathrm{e} 9 \mathrm{rad} / \mathrm{s}$. (a) Quench frequency $f_{q}=1 \mathrm{kHz}$. (b) Quench frequency $f_{q}=1 \mathrm{MHz}$.

\section{Practical analysis method}

From a practical viewpoint, the stability analysis of the solution $\bar{X}_{q}(t)$ is performed by introducing a small-signal 
sinusoidal current source $i_{p}(t)$ at a sensitive location, such as a device node [32]-[36]. Note that unlike ordinary large-signal stability analyses [34]-[36] there are no concerns about frequency commensurability since $i_{p}(t)$ is the only high frequency source and the variables are represented in terms of a single fundamental frequency $\omega$.

The objective is to calculate the LTV transfer function in (11) . With this aim, the frequency $\omega$ of the sinusoidal source $i_{p}(t)$ is swept and, at each sweep step, the linearized envelope-domain system is integrated at the rate of the quench signal. Note that we are integrating a differential equation system in the envelopes $\Delta \bar{X}_{1}(t)$, instead of the full time-domain system. The integration time step should be chosen to properly sample the solution $\bar{X}_{q}(t)$ forced by the quench signal. However, this time step must be sufficiently large to prevent convergence to the oscillatory solution.

The practical stability analysis is carried out through polezero identification [32],[34]-[38]. As stated, the frequency $\omega$ is swept, performing an envelope-domain integration at each frequency step. This provides a sequence of functions $H_{s, m}\left(t, \omega_{i}\right)$, where $i$ represents a counter of the frequency sweep. However, the pole-zero identification is carried at each time value $t_{n}$, where $n$ represents a counter of the time steps. Note that both variables $\omega$ and $t$ are actually discrete and the counter specified above (either $i$ or $n$ ) simply distinguishes the parameter considered in each case. The pole-zero identification is performed by fitting the function $H_{s, m}\left(t_{n}, \omega\right)$ with a quotient of polynomials. Thus, pole-zero identification is applied at each time step $t_{n}$. To achieve the SRO operation, the dynamical poles associated to the non-oscillatory solution must shift to the RHS for a fraction of the quench-signal period $T_{q}$.

The pole-zero identification of $Z(t, \omega)$ provides the timevarying poles under both frozen and non-frozen conditions. These are distinguished by comparing the poles obtained under a time-varying quench-signal, represented versus the time $t$, with those obtained replacing the time-varying quench signal with a dc source depending on an artificial parameter $\eta$. In the case of a frozen behavior, the poles will be overlapped in the two simulations, when traced in terms of $\eta$ and $t$, respectively.

To illustrate this, the function (18) has been identified for the same two values of the quench frequency considered in Fig. 3. In Fig. 4, the real part of the complex-conjugate poles has been traced versus time. For $f_{q}=1 \mathrm{KHz}$, in Fig. 4(a), the poles agree with the ones that would be obtained by making the coefficient $a$ in (16) depend on the artificial parameter $\eta: a(\eta)=A_{d c}+$ $A_{p} \cos \left(\omega_{q} \eta\right)$, represented with diamonds. In the time intervals fulfilling $a(t)+1 / R<0$, the system is unstable, with the complex-conjugate poles at the resonance frequency located on the RHS.

For $f_{q}=1 \mathrm{MHz}$, the circuit behaves in non-frozen conditions since the poles traced versus $t$ are different from those traced (in diamonds) versus $\eta$, as shown in Fig. 4(b). There is an increase in the number of poles, though some of these poles are cancelled with zeroes. The circuit is non-frozen, though the pattern of the non-cancelled poles is still the same as the one in Fig. 4(a). For much higher quench frequencies, the dynamic effects become more relevant and the poles shift to RHS for a shorter interval of the quench period. For $f_{q}=10 \mathrm{MHz}$, in Fig. 4(c), the circuit is stable, as can be easily confirmed with standard time-domain integration.

The increase in the number of poles under non-frozen conditions is due to the discretization of the time variable when numerically solving equation (10). Assuming a BackwardEuler integration rule, applied to (10), the vector of state variables $\Delta \bar{X}_{1}\left(t_{n}\right)$ depends on the input current $I_{p}$ and the vector $\Delta \bar{X}_{1}\left(t_{n-1}\right)$, corresponding to the previous time sample. Recursively proceeding like this, one would obtain an expression of $\Delta \bar{X}_{1}\left(t_{n}\right)$ linearly depending on only $I_{p}$. In this calculation each increment $\Delta \bar{X}_{1}\left(t_{n-k}\right)$ is multiplied by a matrix term involving inversions of frequency- and time-dependent matrixes, as gathered from (10). These inversions of frequency dependent matrixes evaluated at $t_{n}$, to $t_{n-k}$ justify the increment in the number of detected poles. However, the impact of previous points decreases with $k$, as they give rise to higher order effects. One must also take into account that the applied pole-zero identification is based on a fitting of the transfer function $H_{s, m}\left(t_{n}, \omega\right)=\Delta X_{1, m}\left(t_{n}\right) / I_{p}$ with a quotient of polynomials, which is done in a "black-box" way. As the time derivatives become more significant, the transfer function $H_{s, m}\left(t_{n}, \omega\right)$ becomes more complex and a higher polynomial order is required to perform the fitting. This is also consistent with the more significant magnitude variations of $Z(t, \omega)$ in Fig. 3 , as the quench frequency increases.

Under non-frozen conditions, the identification procedure is subject to numerical errors, which are inherent to the time discretization used in the practical computation of the linearized system (10). However, the method is useful for the distinction between frozen and non-frozen behavior, for the determination of the reference times at which the critical pair of complexconjugate poles cross from the LHS to the RHS and vice versa, and for the prediction of stabilization effects. Under sufficient continuity versus the time variable, it will also be applicable to identify the SRO functions, as shown in the next subsection. 


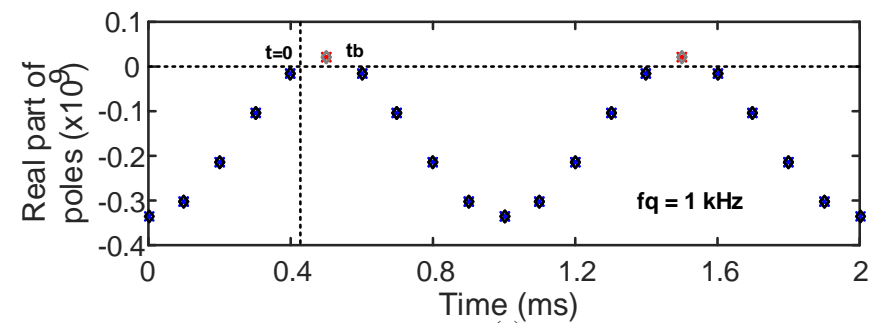

(a)

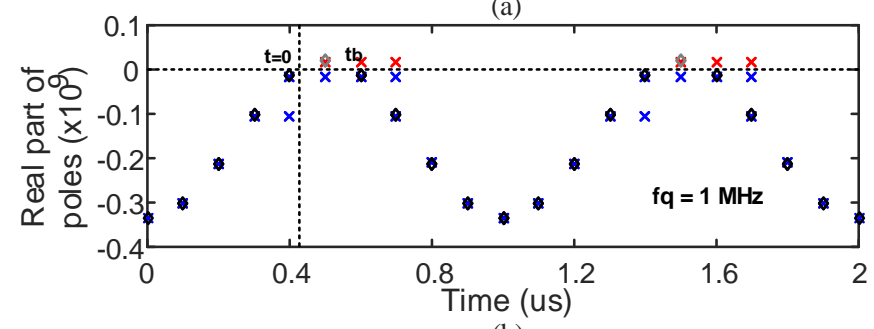

(b)

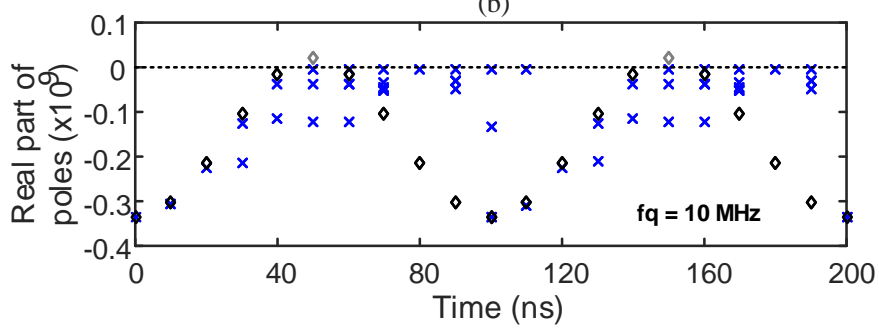

(c)

Fig. 4. Real part of the complex-conjugate poles of the circuit in Fig. 2, traced versus time, for different quench frequencies. The poles obtained through a static analysis in terms of the dummy variable $\eta$ are superimposed, as a reference, in diamonds. (a) $f_{q}=1 \mathrm{kHz}$. (b) $f_{q}=1 \mathrm{MHz}$. (c) $f_{q}=10 \mathrm{MHz}$.

\section{E. Identification of SRO functions}

The SRO functions $p(t), s(t)$ and $K_{\mathrm{s}}$ defined in [2] enable great insight into the SRO operation and lead to an approximate closed-form expression of its input-output relationship. As shown in the following, these functions can be extracted from the pole analysis in the previous sub-section. This will be possible provided that the frequency of the quench signal is not too high to prevent an accurate pole-zero identification.

First, the relevant transition times in the SRO operation are identified from the time variation of the critical pair of complexconjugate poles. The time origin $t=0$ is established at the time value for which the real part of the poles changes from negative to positive (Fig. 4). This reference time approximately constitutes the center of the sensitivity interval [2]. Starting from this time value, the duration of the time interval with the critical poles on the RHS defines the time $t_{b}$, at which the oscillation envelope reaches its maximum amplitude.

At a second stage, the real part of the poles $\sigma(t)$ obtained through the identification of function (11) is fitted with a Fourier series, which can be done due to its periodicity. This is expressed as follows:

$$
\sigma(t)=a_{0}+\sum_{n=1}^{N} a_{n} \cos (n \omega t)+b_{n} \sin (n \omega t)
$$

where $N$ is the number of the harmonic terms used to fit $\sigma(t)$. No more than 4 harmonic terms have been necessary in any of the cases considered in this work. Then, the SRO normalized envelope [2] is calculated with the following expression: $p(t)=\exp \left(\int_{t_{b}}^{t} \sigma(\lambda) d \lambda\right)$

which exhibits a maximum value equal to 1 at $t_{b}$. The integral in the exponent is easily calculated thanks to the Fourier-series expression of $\sigma(t)$.

The above analysis has been applied to the circuit in Fig. 2. Using the poles resulting from the identification in Fig. 3(b), corresponding to the quench frequency $f_{q}=1 \mathrm{MHz}$, one obtains the normalized envelope function $p(t)$ shown in Fig. 5(a). It is compared with the analytical function in [2] and with the oscillatory solution of system (17). To obtain this oscillatory solution, the time step must be sufficiently small to enable the integration of the envelope of the SRO pulse. For this integration, the constant term $I_{p}$ in (17) is replaced with a short small-signal impulse $I_{p} \delta(t-\tau)$, in the envelope scale. The impulse duration should be much shorter than the period of the quench signal. The impulse duration should be chosen to ensure that the integration method recognizes the impulse and does not overpass it. In this particular case the integration time step is $\Delta t=2 \mathrm{~ns}$ and the pulse duration is $2.1 \mathrm{~ns}$. The waveform in Fig. 5(a) actually corresponds to the envelope-domain LTV impulse response of the SRO, evaluated at the time $\tau$. However, provided that $t_{b}$ is not too small in comparison with the quench period $T_{q}$, the integration enables an estimation of the system homogeneous solution. In fact, by just multiplying this envelope by a constant factor, it overlaps with the function $p(t)$.

The results obtained when applying the short impulse at different time instants $\tau$ within the sensitivity interval are shown in Fig. 5(b). This way we are performing a numerical calculation of particular samples of the oscillatory LTV impulse response of the SRO in linear mode. For each impulse, at a given time $\tau$, an envelope-transient simulation is performed at a sufficiently fine time step. A shift in the time value $\tau$ at which the impulse is applied gives rise to a change in the envelope magnitude. In each case, the maximum envelope magnitude is obtained at the time instant $t_{b}$ at which the poles cross from the RHS to the LHS. This is the time value for which the poles have spent the longest on the RHS, so the signal has been able to grow to its maximum amplitude. Due to the relatively large $t_{b}$, the distinct envelopes (resulting from the application of the small impulses) agree with $p(t)$ when simply multiplied by scalar factors.

As a further accuracy evaluation, Fig. 5(c) compares the oscillation pulse obtained through both (20) and the envelope transient method, with the one obtained through standard timedomain integration.

In order to obtain the sensitivity function and the superregenerative gain [2], one can perform several operations on $p(t)$. The sensitivity function can be obtained as follows:

$$
\begin{aligned}
& s(t)=\exp \left(-\int_{0}^{t} \sigma(\lambda) d \lambda\right) \\
& =\exp \left(-\int_{0}^{t_{b}} \sigma(\lambda) d \lambda\right) \exp \left(-\int_{t_{b}}^{t} \sigma(\lambda) d \lambda\right)=\frac{p(0)}{p(t)}
\end{aligned}
$$

And the super-regenerative gain is given by: 


$$
K_{s}=\exp \left(\int_{0}^{t_{b}} \sigma(\lambda) d \lambda\right)=\frac{1}{p(0)}
$$

Then, the system output to a given input signal $v_{i n}(t)$ can be estimated from the approximate input-output relationship derived in [2].

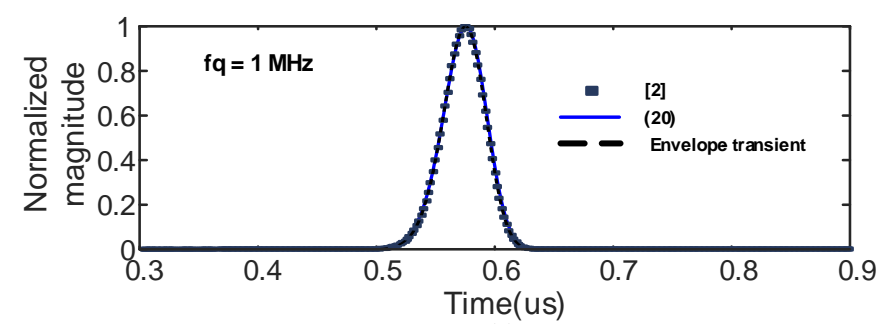

(a)
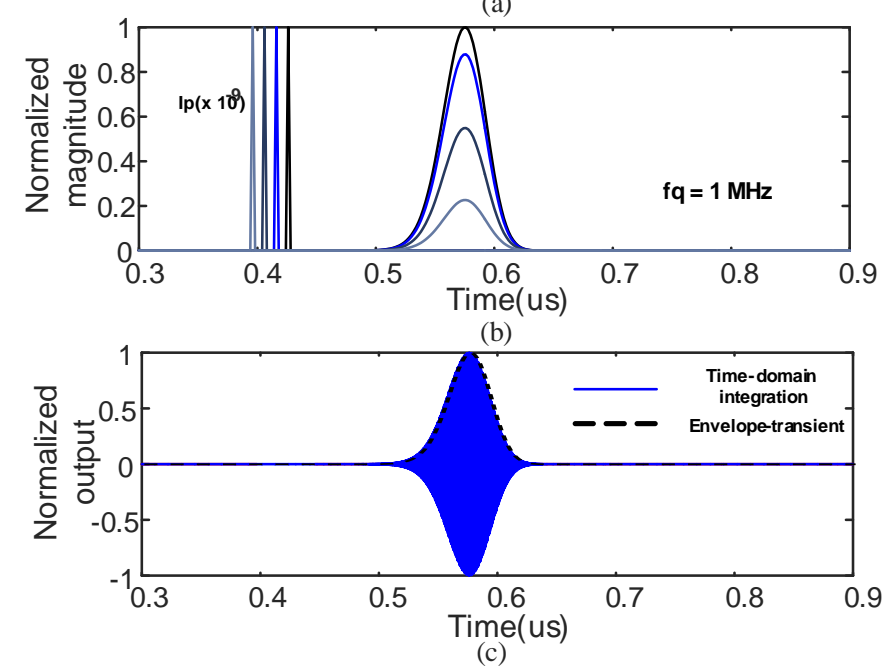

Fig. 5. Response of the SRO in Fig. 2. (a) Normalized envelope function obtained from the real part of the dominant poles $\sigma(t)$, as shown in (20). It is compared with the one given by the analytical expression in [2] and with the results of envelope transient, after the introduction of a small current impulse $I_{p} \delta(t-\tau)$. (b) Application of the impulse at different time instants $\tau$, giving rise to a change in the envelope magnitude. (c) Comparison of the oscillation pulse obtained with time-domain integration and with envelope transient when applying a short-duration sinusoidal signal at the $2.56 \mathrm{GHz}$.

\section{F. Application to a transistor-based oscillator}

As a second example, a transistor-based oscillator at $f_{o}=2.7 \mathrm{GHz}$ is considered. The circuit is shown in Fig. 6. It is based on the transistor NE3210S01 and has been built on Rogers $4003 \mathrm{C}$ substrate $\left(\varepsilon_{\mathrm{r}}=3.55, \mathrm{H}=32\right.$ mils $)$. Complete models are used for the transistor and the transmission lines. Bias capacitors and inductors are considered ideal, since the scattering-parameter models available for these components were ill suited for both envelope-transient analysis and transient simulation.

Fig. 7 shows the variation of the output-oscillation amplitude versus the gate-bias voltage $V_{G G}$ at the drain bias voltage $V_{D D}$ $=0.7 \mathrm{~V}$. As gathered form Fig. 7, the circuit oscillates for $V_{G G}>-0.57 \mathrm{~V}$ in static conditions. The whole oscillation curve is stable.

For the SRO operation, the dc source is replaced with a sinusoidal source of the form: $v_{q}(t)=V_{d c}+V_{p} \cos \left(\omega_{q} t\right)$, where $V_{d c}=-1.584 \mathrm{~V}$ and $V_{p}=1.06 \mathrm{~V}$. The stability properties, under this quench signal, are analyzed using (11). With this aim, a small-signal current source $i_{p}(t)$ is connected in parallel with the drain terminal. Fig. 8(a) and (b) present the time variation of the real part of the dominant pair of complex-conjugate poles at the oscillation frequency for two different values of the quench frequency: $f_{q 1}=1 \mathrm{kHz}$ and $f_{q 2}=8 \mathrm{MHz}$. In both figures, the real part of the poles has been traced versus time. The poles obtained in static conditions have also been represented (with diamonds), as a reference. These poles have been calculated by replacing the sinusoidal quench-signal source with a DC source, expressed as: $v_{q}(\eta)=V_{d c}+V_{p} \cos \left(\omega_{q} \eta\right)$. For $f_{q 1}=1 \mathrm{kHz}$ [Fig. $8(\mathrm{a})$ ], the circuit behaves as frozen, since the poles are overlapped with the "static" ones. For $f_{q 2}=8 \mathrm{MHz}$ [Fig. 8(b)], the pattern is more complex, with the numerical increase in the number of poles that is characteristic of the non-frozen behavior. The most significant effect is the positive time shift of the unstable time interval, corresponding to a delay with respect to the static situation. To validate this result, the circuit is excited with an input source at $\omega$, providing a short impulse $v_{\text {pulse }}(t, \tau)$ in the envelope time scale. This impulse is applied at a time instant $\tau$ within the sensitivity interval, and an envelopetransient simulation of the transistor-based SRO is carried out. The magnitude of the envelope of the output signal is shown in Fig. 8(c). Fig. 8(d) presents an expanded view that allows comparing the envelope magnitude with the variation of the static poles and the poles predicted through (11). The time $t_{b}$ at which the envelope exhibits its maximum magnitude should agree with the time at which the poles cross from the RHS to the LHS. This maximum is well predicted by the poles obtained from (11), whereas the static poles cross at a smaller time value $t<t_{b}$.

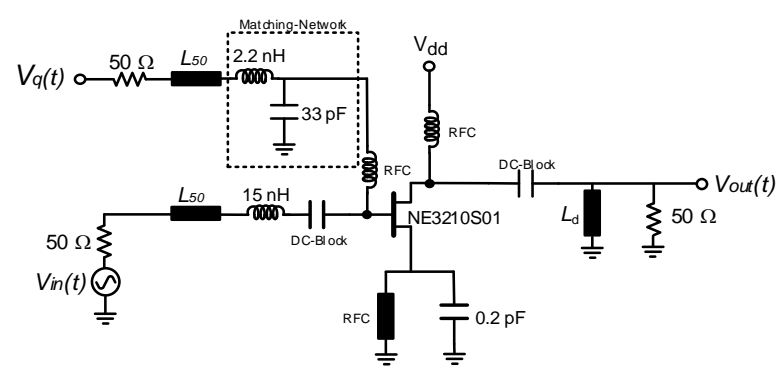

Fig. 6. Transistor-based oscillator. It is based on the transistor NE3210S01 and has been built on Rogers $4003 \mathrm{C}$ substrate $(\varepsilon \mathrm{r}=3.55, \mathrm{H}=32 \mathrm{mils})$. The oscillation at the frequency $f_{o}=2.7 \mathrm{GHz}$ is switched on and off through a sinusoidal quench signal, introduced in the gate bias line.

Fig. 9 shows the SRO response to small-signal voltage impulses injected at distinct time values within the sensitivity period. Two different values of $V_{d c}$ have been considered: $V_{d c}=-1.564 \mathrm{~V}$ in Fig. 9(a), and $V_{d c}=-1.584 \mathrm{~V}$ in Fig. 9(b), with $V_{p}=1.06 \mathrm{~V}$ in the two cases. The simulations have been carried out exciting the circuit with an input impulse $v_{\text {pulse }}(t, \tau)$

and performing an envelope transient simulation for each $\tau$. The solutions should correspond to the oscillatory LTV impulse response of the transistor-based SRO in linear mode, when evaluated at the particular $\tau$ values. In the case of $V_{d c}=-1.564$ $\mathrm{V}$, the sensitivity period and the envelope are relatively distant, 
so the solution envelope should approach that of the homogeneous oscillatory solution. However, the solution envelope is slightly altered due to the effect of the applied impulse. This effect is more significant for $V_{d c}=-1.584 \mathrm{~V}$, in Fig. 9(b). In this case, the poles are on the RHS only for a short time interval in comparison with the quench period $T_{q}$. As a result, the sensitivity interval is too close to the oscillationenvelope maximum, which prevents an accurate identification of the normalized envelope $p(t)$ through envelope-transient simulations. The envelope response first decays and then grows because the pulses are inserted before the poles cross to the RHS. A general numerical methodology to characterize the SRO behavior in linear mode will be presented in the next section. It enables a global investigation of the SRO response and should be usable to develop an accurate behavioral model, applicable in system-level simulations.

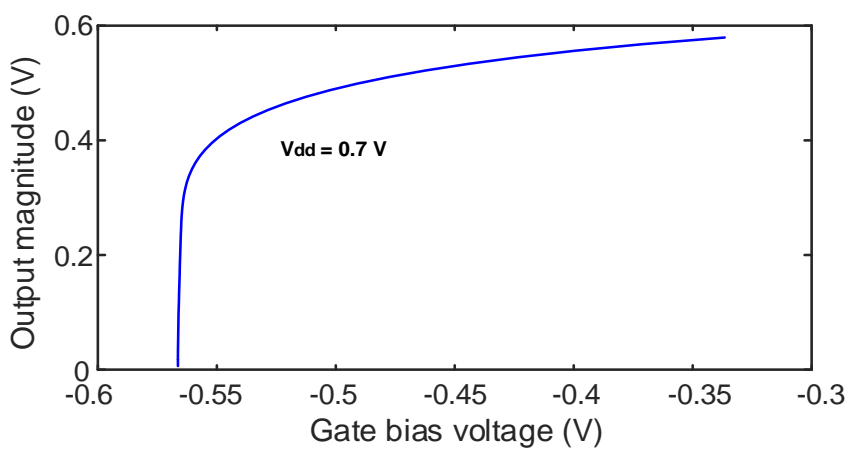

Fig. 7. Variation of the output-oscillation magnitude versus the gate-bias voltage $V_{G G}$ for the drain bias voltage $V_{D D}=0.7 \mathrm{~V}$. The circuit oscillates for $V_{G G}>-0.57 \mathrm{~V}$ in static conditions.

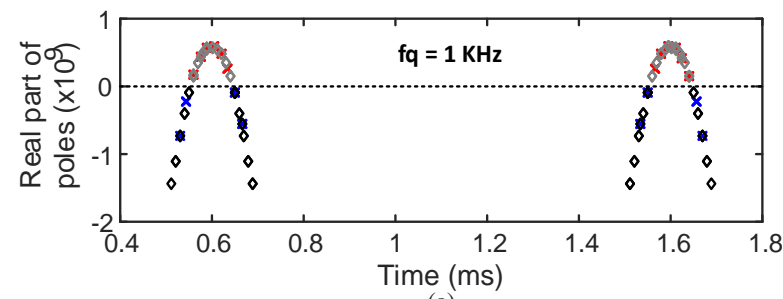

(a)

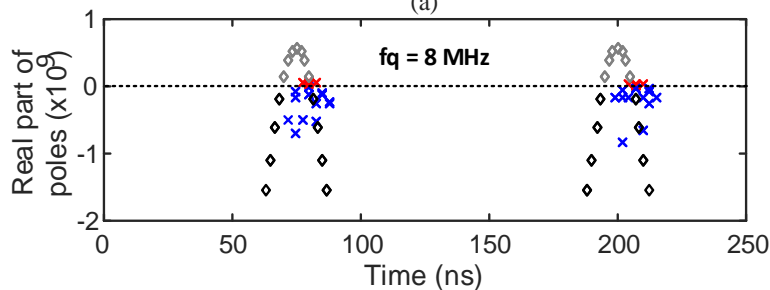

(b)
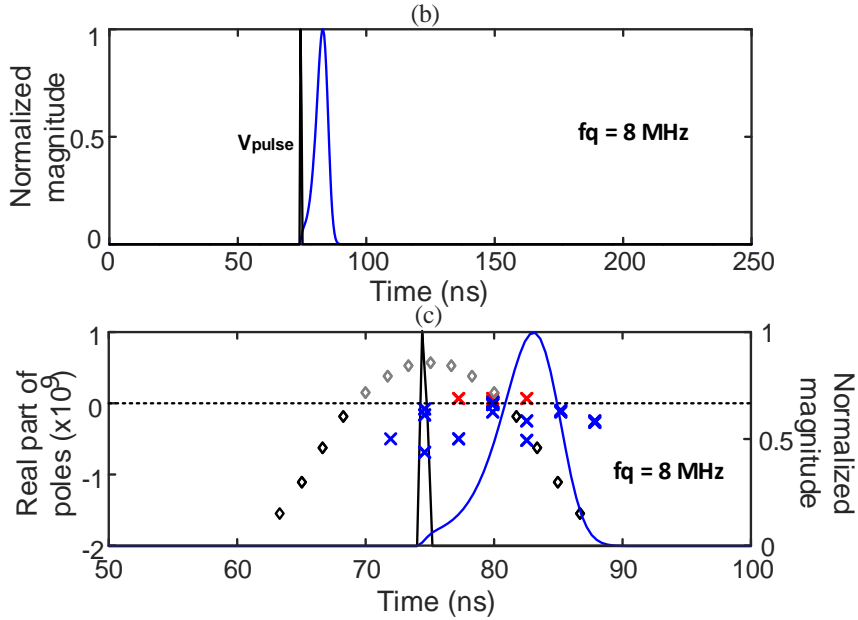

(d)

Fig. 8. Stability analysis of the transistor-based oscillator, under the quench signal $v_{q}(t)=V_{d c}+V_{p} \cos \left(\omega_{q} t\right)$, where $V_{d c}=-1.584 \mathrm{~V}$ and $V_{p}=1.06 \mathrm{~V}$ for two different values of the quench frequency $\omega_{q}$. The poles obtained through a static analysis in terms of the dummy variable $\eta$ are superimposed, as a reference, in diamonds. (a) $f_{q}=1 \mathrm{kHz}$. (b) $f_{q}=8 \mathrm{MHz}$. (c) Validation through an envelopetransient analysis for $f_{q}=8 \mathrm{MHz}$. (d) Expanded view comparing the variations of the static poles and the poles predicted through (11) with the magnitude of the envelope resulting from the application of an impulse $v_{\text {pulse }}(t, \tau)$.
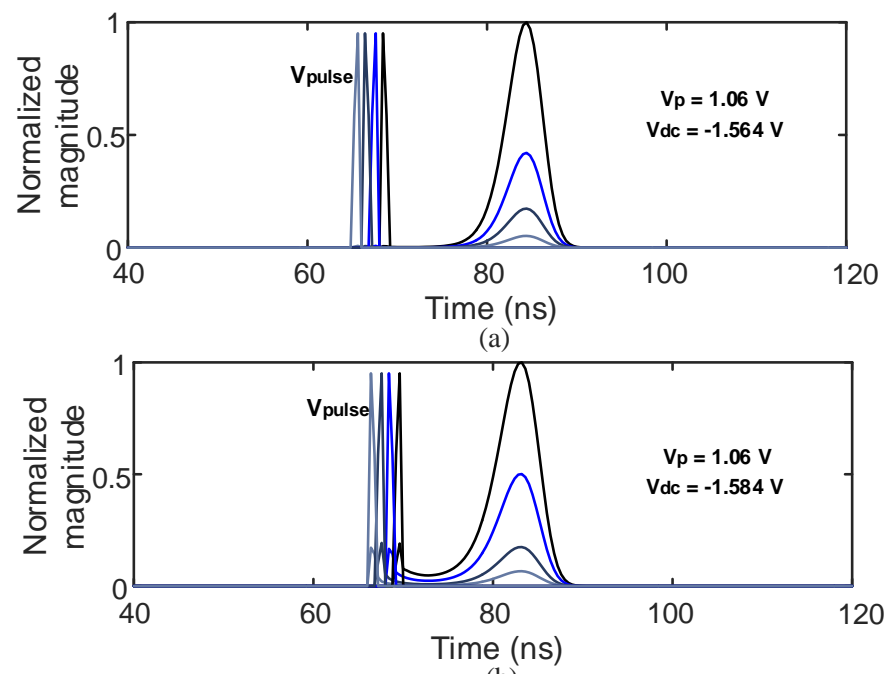

(b)

Fig. 9. Response of the transistor-based SRO to impulses injected at distinct time values within the sensitivity period, calculated with circuit-level envelopetransient simulations. Two different values of $V_{d c}$ are considered, with $V_{p}=1.06 \mathrm{~V}$ and $f_{q}=8 \mathrm{MHz}$. (a) $V_{d c}=-1.564 \mathrm{~V}$. (b) $V_{d c}=-1.584 \mathrm{~V}$. 


\section{OSCILLATORY LTV IMPULSE RESPONSE OF THE SRO IN LINEAR MODE}

An envelope-domain LTV impulse response of the SRO in oscillatory regime is derived in this section. The time-domain impulse response is denoted $h(t, \tau)$, where $\tau$ indicates the instant when the impulse is applied. The envelope-domain impulse response is denoted as $\tilde{h}(t, \tau)$. Lower case is used for full timedomain variables and capitals are used for envelope-domain variables.

\section{A. Formal derivation of the oscillatory LTV impulse response}

From LTV system theory [23]-[25], the output under an arbitrary small-signal input $v_{i n}(t)$ is given by:

$v_{\text {out }}(t)=\int_{-\infty}^{\infty} h(t, \tau) v_{\text {in }}(\tau) d \tau$

The LTV transfer function associated with $h(t, \tau)$ is calculated as:

$$
H(t, \omega)=\int_{-\infty}^{\infty} h(t, \tau) e^{-j \omega(t-\tau)} d \tau
$$

Then, the output signal can be obtained from [23]-[25]:

$$
v_{\text {out }}(t)=\frac{1}{2 \pi} \int_{-\infty}^{\infty} H(t, \omega) v_{\text {in }}(\omega) e^{j \omega t} d \omega
$$

Where $v_{i n}(\omega)$ is the Fourier spectrum of the time-domain signal $v_{i n}(t)$. In the envelope domain, the aim will be to get a relationship between the Fourier transform of the low pass equivalent $v_{i n}(t)$, denoted as $V_{i n}(\omega)$, and the low-pass equivalent of the output signal $V_{\text {out }}(t)$. The spectrum of $v_{\text {in }}(t)$ is given by $V_{i n}\left(\omega-\omega_{p}\right)+V_{i n}^{*}\left(\omega+\omega_{p}\right)$, where the particular carrier frequency $\omega_{p}$ has been considered. Replacing this expression into (25), one obtains:

$v_{\text {out }}(t)=\frac{1}{2 \pi} \int_{-\infty}^{\infty} H(t, \omega)\left[V_{\text {in }}\left(\omega-\omega_{p}\right)+V_{\text {in }}^{*}\left(\omega+\omega_{p}\right)\right] e^{j \omega t} d \omega$

Next, the change of variables $\omega=\Omega+\omega_{p}$ and $\omega=\Omega^{\prime}-\omega_{p}$ is used in (26):

$$
\begin{aligned}
& v_{\text {out }}(t)=\frac{e^{j \omega_{p} t}}{2 \pi} \int_{-B / 2}^{B / 2} H\left(t, \Omega+\omega_{p}\right) V_{\text {in }}(\Omega) e^{j \Omega t} d \Omega+ \\
& \frac{e^{-j \omega_{p} t}}{2 \pi} \int_{-B / 2}^{B / 2} H\left(t, \Omega^{\prime}-\omega_{p}\right) V_{i n}^{*}\left(\Omega^{\prime}\right) e^{j \Omega^{\prime} t} d \Omega^{\prime}
\end{aligned}
$$

where $B$ is the analysis bandwidth, about the reference frequency $\omega_{p}$. Representing the output signal in terms of its low pass equivalent: $v_{\text {out }}(t)=V_{\text {out }}(t) e^{j \omega_{p} t}+V_{\text {out }}^{*}(t) e^{-j \omega_{p} t}$, one derives the following envelope-domain relationship:

$$
V_{\text {out }}(t)=\frac{1}{2 \pi} \int_{-B / 2}^{B / 2} H\left(t, \Omega+\omega_{p}\right) V_{\text {in }}(\Omega) e^{j \Omega t} d \Omega
$$

To obtain the LTV impulse response, it is taken into account that the Fourier transform of an impulse applied at time $\tau$ is $e^{-j \Omega \tau}$. Thus, the envelope-domain LTV impulse response, denoted as $\tilde{h}(t, \tau)$, is obtained from

$$
\tilde{h}(t, \tau)=\frac{1}{2 \pi} \int_{-B / 2}^{B / 2} H\left(t, \Omega+\omega_{p}\right) e^{j \Omega(t-\tau)} d \Omega
$$

In the following, a methodology for the practical calculation of $\tilde{h}(t, \tau)$ is provided.

\section{B. Practical analysis methodology}

The numerical procedure for the practical calculation of (29) is composed of two steps, described in the following and shown in the algorithm of Table II. The code has been written in MATLAB.

\section{Step 1. Calculation of the transfer function $H(t, \omega)$}

For the calculation of $H(t, \omega)=H\left(t, \Omega+\omega_{p}\right)$, one should replace the original circuit input with a small-signal sinusoidal source $v_{\text {in }}(t)$, at the high-frequency frequency $\omega$. In the positive spectrum, the sinusoidal input is represented as $V_{i n} e^{j \omega t}$, where $V_{i n}$ is a constant value. The function $H(t, \omega)$ is obtained as the ratio between the output-signal envelope at $\omega$, expressed as $V_{\text {out }}(t, \omega)$, and $V_{i n}$. That is:

$H(t, \omega)=\frac{V_{\text {out }}(t, \omega)}{V_{\text {in }}}$

To calculate the LTV transfer function $H(t, \omega)$ in $(30)$, one sets the magnitude of the sinusoidal input source to the low level that should be used in the practical application. This is because due to the oscillation quenching there can be a high sensitivity to the input signal and the possible nonlinear behavior at the application input power should be detected at this stage. Indeed, quench signals leading to a logarithmic operation mode are easily identified at this stage and should be discarded.

To calculate (30) the frequency $\omega$ of the input sinusoidal source $v_{i n}(t)$ is swept in the interval $\left(\omega_{\min }, \omega_{\max }\right)$ and, at each sweep step, an envelope-domain integration is carried out. This will provide the double entry function $H\left(t_{n}, \omega_{i}\right)$, where $n$ goes from 1 to the maximum number of time samples $N$ and $i$ is a counter of the frequencies considered in the sweep, covering the interval $\left(\omega_{\min }, \omega_{\max }\right)$. The time values $t_{1}$ to $t_{N}$ should be identical for each frequency $\omega_{i}$. The sampling time step $\Delta t$ must be small enough to enable the oscillation start-up in all cases, though overlapping bandwidths must be avoided to ensure the validity of the low-pass representation. Therefore the total bandwidth in the frequency sweep must fulfil $B=f_{\max }-f_{\min }>2 / \Delta t$, where $f=\omega /(2 \pi)$.

Regarding the frequency sweep in the interval $\left(\omega_{\min }, \omega_{\max }\right)$, it is convenient to use a higher concentration of frequency samples about the resonance frequency, which is done by reducing the frequency step in the middle region through a suitable sweep plan. This central frequency interval with more significant variations of the transfer function is easily determined through a simple inspection of these transfer functions.

The function $H(t, \omega)$ fully agrees with $H\left(t, \Omega+\omega_{p}\right)$, since $\omega_{p}$ is just a shift with respect to the baseband frequency $\Omega$. It is well suited to make $\omega_{p}$ agree with the central frequency of the SRO resonance interval. The function $H\left(t, \Omega+\omega_{p}\right)$ is obtained by simply replacing the original frequency column with $\Omega=\omega$ $-\omega_{p}$. The double entry function $H\left(t, \Omega+\omega_{p}\right)$ is exported form the envelope-transient circuit-level simulator and read in the inhouse software. 
As an additional comment, both (11) and (30) are LTV transfer functions, but they are conceptually very different. The function (11), used for the stability analysis, is obtained by linearizing the SRO about the non-oscillatory periodic solution $\bar{X}_{q}(t)$, forced by the quench signal. The input considered in (11) is a small-signal sinusoidal current source $i_{p}(t)$, introduced at a sensitive node. This is because the purpose of this transfer function is to analyze the stability variations of the nonoscillatory solution under the effect of the quench signal, so we need controllability and observability [36]. Otherwise unstable poles might be numerically cancelled with RHS zeroes. Instead, function (30) is obtained linearizing the SRO about the oscillatory solution $\bar{X}_{o}(t)$. The input considered in (30) is a small-signal sinusoidal source $v_{i n}(t)$, introduced at the location of the input signal to be amplified.

The above methodology has been applied to the transistorbased oscillator in Fig. 6, under a sinusoidal quench signal of the form $v_{q}(t)=V_{d c}+V_{p} \cos \left(\omega_{q} t\right)$. The amplitude of the input sinusoidal signal is $V_{i n}=0.1 \mathrm{mV}$. The integration time step is $\Delta t=1 \mathrm{~ns}$.

Representing the magnitude and phase of the low-pass equivalent $H\left(t, \Omega+\omega_{p}\right)$ versus $\Omega$ one obtains the set of transfer functions in Fig. 10. Each trace corresponds to a different time value in the envelope-transient integration interval, given by (50 ns, $600 \mathrm{~ns}$ ), with the time step 1 ns. Fig. 10(a) and (b) are obtained for $V_{d c}=-1.584 \mathrm{~V}$ and $V_{p}=1.06 \mathrm{~V}$. Fig. 10 (a) presents the magnitude of the resonance curves, whereas Fig. 10(b) presents the phase of these curves. Fig. 10(c) shows the magnitude of the resonance curves obtained for $V_{d c}=-1.56 \mathrm{~V}$, which evidence a nonlinear behavior. A quantitative detection of the nonlinear mode is carried out by just inspecting the harmonic content, at multiples of the oscillation frequency, that is, by evaluating $\left|V_{\text {out }, k}(t)\right| / V_{\text {in }}$.

Once the double entry function $H\left(t_{n}, \Omega_{\mathrm{i}}+\omega_{p}\right)$ is available in the in-house software, the impulse response $\tilde{h}(t, \tau)$ is calculated, for each $\tau$, by means of a loop in the time variable $t_{n}$ (going from $t_{1}$ to $t_{N}$ ), as described next.
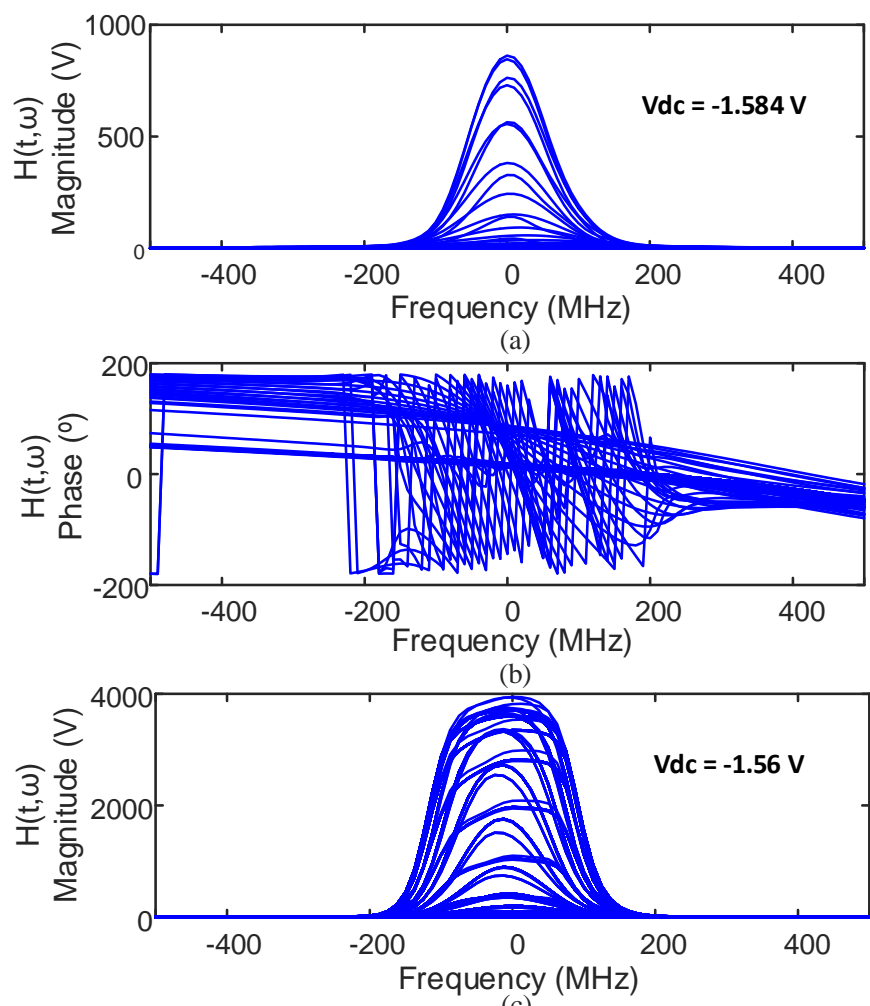

(c)

Fig. 10. Function $H(t, \omega)$ corresponding to the transistor-based oscillator in Fig. 6. The quench frequency is $f_{q}=8 \mathrm{MHz}$ and $V_{p}=1.06 \mathrm{~V}$. Each trace corresponds to a different time value in the envelope-transient integration interval, given by $(50 \mathrm{~ns}, 600 \mathrm{~ns})$, with the time step $1 \mathrm{~ns}$. (a) Magnitude of $H(t, \omega)$ for $V_{d c}=-1.584$ V. (b) Phase of $H(t, \omega)$ for $V_{d c}=-1.584 \mathrm{~V}$. (c) Magnitude of $H(t, \omega)$ for $V_{d c}=$ $-1.56 \mathrm{~V}$.

\section{Step 2. Loop in $t_{n}$ for the calculation of $\tilde{h}(t, \tau)$}

The usual inverse Fourier transform is applied to a function depending only on frequency. However, the function $H(t, \omega)=H\left(t, \Omega+\omega_{p}\right)$ in (30) also depends on the time $t$. Our way to deal with this additional dependence is to parametrize the time $t$, which, as indicated, is discretized in the samples $t_{1}$, $t_{n}, \ldots t_{N}$. At each time $t_{n}$, the corresponding value $\tilde{h}\left(t_{n}, \tau\right)$ of the LTV impulse response $\tilde{h}(t, \tau)$ is calculated as:

$$
\tilde{h}\left(t_{n}, \tau\right)=\frac{1}{2 \pi} \int_{\omega_{\min }-\omega_{p}}^{\omega_{\max }-\omega_{p}} H\left(t_{n}, \Omega+\omega_{p}\right) e^{j \Omega\left(t_{n}-\tau\right)} d \Omega
$$

The whole function $\tilde{h}(t, \tau)$ for a particular $\tau$ is given by a sequence of time values $\tilde{h}\left(t_{n}, \tau\right)$, where $t_{1}, t_{n}, \ldots t_{N}$.

The above method has been applied to calculate the envelope-domain LTV impulse response of the transistor-based SRO of Fig. 6. In Fig. 11 the results are compared with those obtained through circuit-level envelope-transient simulations, where several short-time impulses are applied at different time values $\tau$. To estimate the LTV impulse response through envelope-transient simulations, an input pulse has to be introduced in the circuit at the time $\tau$. This involves an actual realization of the envelope-domain impulse and a circuit-level simulation for each $\tau$ value. Instead, the new LTV impulse is obtained by simply performing a sequence of IFT under the variation of $\tau$, since all the information on the SRO response is 
contained in the oscillatory LTV transfer function. For the specific $\tau$ values considered in Fig. 11, the results of (30)-(31) should agree with those provided by envelope-transient simulations, as validated in that figure.

$$
\text { TABLE II }
$$

ALGORITHM FOR THE CALCULATION OF THE LTV IMPULSE RESPONSE $\tilde{h}(t, \tau)$

\begin{tabular}{|c|c|}
\hline Read Time_vector & $\begin{array}{l}\text { Reading of the vector of time } \\
\text { values exported from ADS }\end{array}$ \\
\hline Read Frequency_vector & $\begin{array}{l}\text { Reading of the frequency } \\
\text { vector exported from ADS. } \\
\text { Prior to exporting, this } \\
\text { frequency vector is translated } \\
\text { doing } f=f_{\text {sweep }}-f_{p} \text {, where } f_{\text {sweep }} \\
\text { are the frequencies in the } \\
\text { sweep. }\end{array}$ \\
\hline$f_{X}=$ Frequency_vector & $\begin{array}{l}\text { Definition of the frequency } \\
\text { vector }\end{array}$ \\
\hline $\begin{array}{l}T=\max (\text { Time_vector) - } \\
\min (\text { Time_vector) }\end{array}$ & $\begin{array}{l}\text { Calculation of the duration of } \\
\text { the time interval }\end{array}$ \\
\hline $\begin{array}{l}N= \\
\text { length(Time_vector) }\end{array}$ & $\begin{array}{l}\text { Calculation of the number of } \\
\text { time points }\end{array}$ \\
\hline $\begin{array}{l}\text { tau }= \\
\text { Iinspace (taul, tau2, tau } \\
\text { _points) }\end{array}$ & $\begin{array}{l}\text { Definition of the vector of tau } \\
\text { values considered in } \tilde{h}(t, \tau) \text {. } \\
\text { (The interval is tau1, tau } 2 \text { ) }\end{array}$ \\
\hline for $k=1$ : length (tau) & $\begin{array}{l}\text { Beginning of the loop that } \\
\text { calculates a sequence of } \\
\text { functions } \tilde{h}(t, \tau) \text {, one for } \\
\text { each } \tau\end{array}$ \\
\hline for $n=1: N$ & $\begin{array}{l}\text { Beginning of the loop that } \\
\text { calculates } \tilde{h}(t, \tau)\end{array}$ \\
\hline $\begin{array}{l}\operatorname{Exp} w \operatorname{tau}=\exp (- \\
\left.1 i * 2 * p i * f_{x} * \operatorname{tau}(k)\right)\end{array}$ & $\begin{array}{l}\text { Definition of the exponential } \\
\text { function } e^{-j \Omega \tau}\end{array}$ \\
\hline Read $f, H\left(t_{n}, f\right)$ & $\begin{array}{l}\text { Reading of the function } \\
f, H\left[t_{n}, 2 \pi\left(f+f_{p}\right)\right] \\
\text { exported from ADS }\end{array}$ \\
\hline $\begin{array}{l}h(n)=I F T(t n, H . * \\
\text { Exp_wtau) }\end{array}$ & $\begin{array}{l}\text { Calculation of the following } \\
\text { IFT integral: } \\
\tilde{h}\left(t_{n}, \tau\right)= \\
\frac{1}{2 \pi} \int_{\omega_{\min }-\omega_{p}}^{\omega_{\max }-\omega_{p}} H\left(t_{n}, \Omega+\omega_{p}\right) e^{j \Omega\left(t_{n}-\tau\right)} d \Omega\end{array}$ \\
\hline end & \\
\hline
\end{tabular}

Fig. 12 shows the magnitude of the output-signal envelope, obtained when sweeping the time $\tau$ in (30)-(31). The whole analysis in Fig. 12, using (30)-(31), takes $12.18 \mathrm{~s}$ in a computer with $16 \mathrm{~GB}$ RAM, intel ${ }^{\circledR} \mathrm{Core}^{\mathrm{TM}} \mathrm{i} 7-6700 \mathrm{CPU}$ at $3.4 \mathrm{GHz}$. This analysis is much more complete than the one in Fig. 11, which only considers four input pulses. Note that the pulses in Fig. 12 are represented only for illustration purposes, since (30)-(31) directly provides the LTV impulse response at each particular $\tau$ value. These impulses, obtained through the IFT of $e^{-j \Omega \tau}$, have also been represented in Fig. 12. Many $\tau$ values have been considered due to the negligible computational cost.

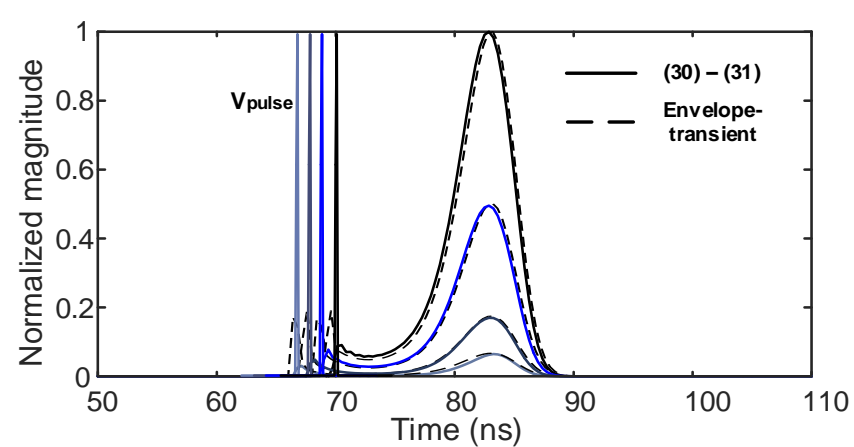

Fig. 11. Transistor-based SRO. Comparison between LTV impulse responses obtained through circuit-level envelope-transient simulations (Fig. 9(b)) and with the numerical method in (30)-(31), departing from the transfer-function in Fig. 10(a) and Fig.10(b).

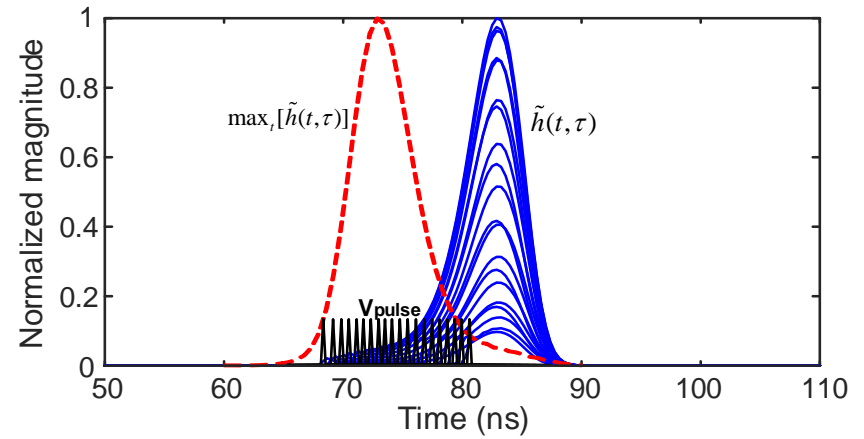

Fig. 12. Transistor based SRO. Normalized magnitude of the output-voltage envelope when sweeping the time $\tau$ in (30)-(31). The sensitivity interval has been considered as the one comprised between the $\tau$ values at which the maximum envelope magnitude is $0.1 V_{\max }$, where $V_{\max }$ is the maximum magnitude obtained when sweeping $\tau$ through the whole interval. This interval is $(68 \mathrm{~ns}, 80 \mathrm{~ns})$. The maximum sensitivity is obtained at $\tau=74 \mathrm{~ns}$. The sensitivity interval has also been calculated by evaluating the maximum of the oscillatory LTV impulse response $\tilde{h}(t, \tau)$, in terms of the time $t$, under variation of the impulse time $\tau$, in dashed line. The whole analysis takes $12.18 \mathrm{~s}$ in a computer with $16 \mathrm{~GB}$ RAM, intel ${ }^{\circledR}$ Core $^{\mathrm{TM}} \mathrm{i} 7-6700 \mathrm{CPU}$ at $3.4 \mathrm{GHz}$.

\section{Comparison with previous SRO models}

The LTV impulse response fully characterizes the SRO behavior under a given quench signal. The reference time $t=0$ is taken from the middle time of the sensitivity interval. This is calculated by evaluating the maximum of the oscillatory LTV impulse response $\tilde{h}(t, \tau)$, in terms of the time $t$, under variations of the impulse time $\tau$. This maximum, expressed as $\max _{t}[\tilde{h}(t, \tau)]$, is represented versus $\tau$, which in the transistorbased oscillator gives rise to the dashed-line curve of Fig. 12(a). This calculation is straightforward using (30)-(31) but would be very demanding when based on a sequence of circuit-level envelope transient simulations.

If an equivalence with the conventional model is wished, the normalized envelope $p(t)$ can be approximated by the impulse response $\tilde{h}(t, \tau)$, normalized to 1 . The normalized envelope $p(t)$ is the homogeneous solution of the SRO, whereas $\tilde{h}(t, \tau)$ is calculated under an impulse excitation, so there is a certain error 
here. However, the functions $\tilde{h}(t, \tau)$ have approximately the same shape except near the impulse time $\tau$. In fact, when varying $\tau$ through the sensitivity interval, there is a scaling effect of the output signal, this meaning that they approximately overlap when multiplied by suitable constant scalars (except near the impulse time $\tau$ ). The super-regenerative gain can be obtained from $p(0)$, as shown in equation (22). The constant factor $K_{0} \xi_{0}$ of [2] can be obtained through fitting.

In Fig, 12 the sensitivity interval is (68 ns, 80ns), centered about $\tau=74 \mathrm{~ns}$. The maximum amplitude of the output pulse is obtained when injecting the impulse at $\tau=74 \mathrm{~ns}$. The results are also consistent with the predictions through pole-zero identification in Fig. 8.

\section{Hang-over effects}

The method is able to predict the hang-over effects. However, it is applicable to SROs in the linear operation. With the transistor-based SRO, we did not observe hang-over in linear mode, only in logarithmic mode. With the negative-resistor oscillator in Fig. 2 it was possible to observe hang-over effects when replacing the sinusoidal quench signal with a sawtooth signal. Fig. 13(a) presents the sawtooth quench signal. Fig. 13(b) shows the oscillatory LTV transfer function $H\left(t, \Omega+\omega_{p}\right)$ traced versus $\Omega$ for different $t$ values in a time interval comprising 5 quench periods, given by $(0,1 \mu \mathrm{s})$, with the time step $1 \mathrm{~ns}$. At some $t$ values, the magnitude of $H\left(t, \Omega+\omega_{p}\right)$ exhibits several local maxima, which is indicative of hang-over effects [1], [2]. Next, a short-duration sinusoidal signal has been applied at the time 50 ns. Fig. 13(c) compares the output signal obtained with (30)-(31) and with standard time-domain integration. As can be seen, there is a very good agreement. Fig. 13(d) presents an expanded view of the first output pulse. The result is successfully compared with standard time-domain integration.

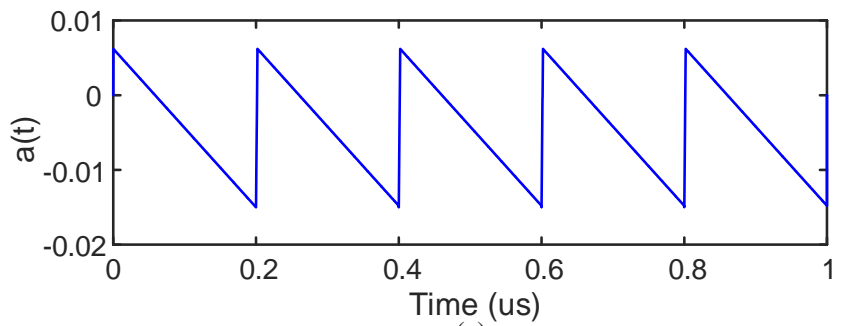

(a)
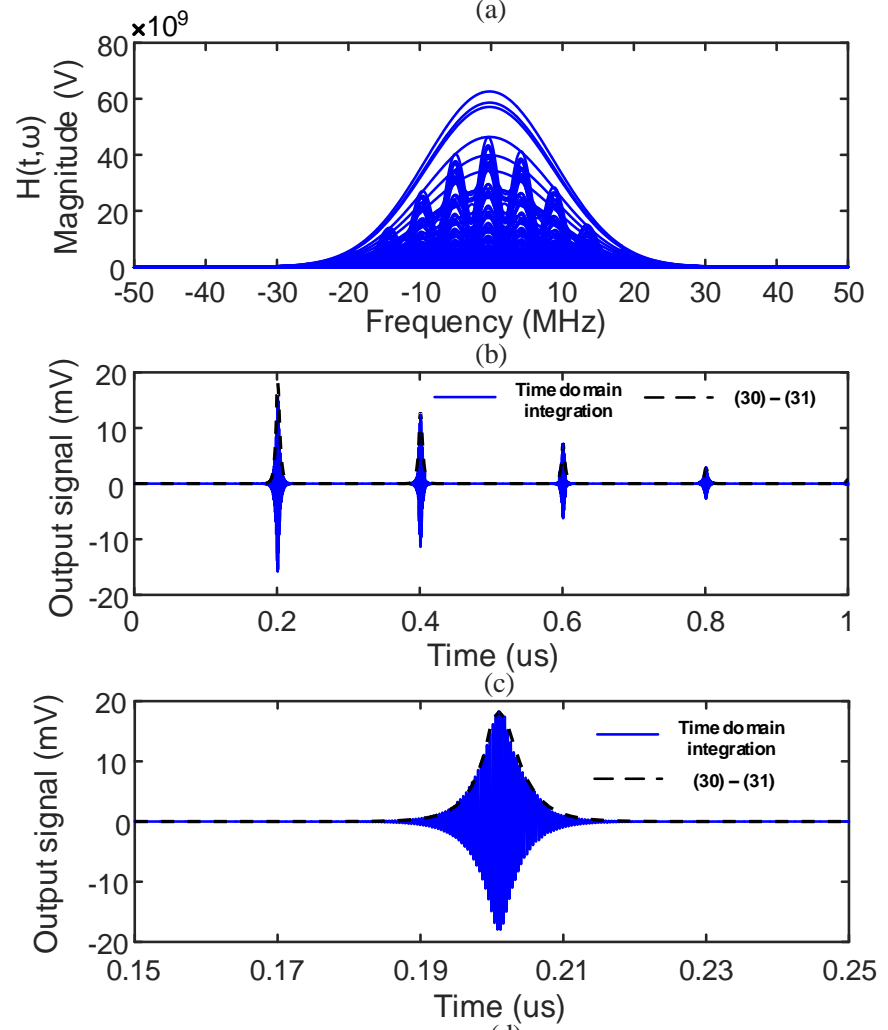

(d)

Fig. 13. Prediction of hang-over effects. (a) Sawtooth quench signal. (b) Oscillatory LTV transfer function $H(t, \omega)$ traced versus $\omega$ for different $t$ values in a time interval comprising 5 quench periods. (c) Output pulses obtained with $H(t, \omega)$ and with standard time-domain integration. (d) Expanded view of the first output pulse. The result is compared with standard time-domain integration.

\section{OUTPUT SIGNAL UNDER AN ARBITRARY INPUT MODULATION}

Let a modulated input signal be considered. It will be expressed in terms of its low pass equivalent as: $v_{i n}(t)=2 \operatorname{Re}\left[V_{i n, \omega}(t) e^{j \omega t}\right]$. For convenience, this low-pass equivalent will be redefined with respect to $\omega_{p}$, by doing: $V_{i n}(t)=V_{i n, \omega}(t) e^{j\left(\omega-\omega_{p}\right) t}$. Then, the output signal is obtained from the LTV transfer function by means of the following integral:

$V_{\text {out }}(t)=\frac{1}{2 \pi} \int_{-B / 2}^{B / 2} H\left(t, \Omega+\omega_{p}\right) V_{\text {in }}(\Omega) e^{j \Omega t} d \Omega$

The time interval and number of samples must be the same in $V_{i n}(t)$ and $H\left(t, \Omega+\omega_{p}\right)$, which is directly handled in the inhouse software. Remember that $H\left(t, \Omega+\omega_{p}\right)$ is obtained through (30) by means of a frequency sweep, applied to a smallsignal sinusoidal input $V_{i n} e^{j \omega t}$. If the carrier frequency $\omega$ does 
not agree with $\omega_{p}$, the low pass equivalent calculated with (32) will be $V_{\text {out }}(t)=V_{\text {out }, \omega}(t) e^{j\left(\omega-\omega_{p}\right) t}$.

In a manner similar to what was described in the previous sub-section, once the double entry function $H\left(t_{n}, \Omega_{i}+\omega_{p}\right)$ is available in the in-house software, the output envelope $V_{\text {out }}(t)$ is calculated by means of a loop in the time variable $t_{n}$ (going from $t_{1}$ to $\left.t_{N}\right)$. At each time step, the following IFT integral function is calculated:

$V_{\text {out }}\left(t_{n}\right)=\frac{1}{2 \pi} \int_{\omega_{\min }-\omega_{p}}^{\omega_{\max }-\omega_{p}} H\left(t_{n}, \Omega+\omega_{p}\right) V_{\text {in }}(\Omega) e^{j \Omega t_{n}} d \Omega$

The whole function $V_{\text {out }}(t)$ is given by the sequence of time values $V_{\text {out }}\left(t_{n}\right)$, where $t_{1}, t_{n}, \ldots t_{N}$.

Thus, once the LTV transfer function $H\left(t, \Omega+\omega_{p}\right)$ is available, the output signal under any arbitrary input is calculated through the integral expression (33). To clarify the various steps of the procedure, the algorithm has been summarized in Table III.

For illustration, the method has been tested under an on-off keying modulation and a QPSK modulation. In the two cases, the LTV transfer function in Fig. 10(a) and Fig. 10(b) will be used for the calculation of the output signal.

Initially, an on-off keying modulation at the carrier frequency $f=2.7 \mathrm{GHz}$, with the modulation frequency $f_{\text {mod }}=800 \mathrm{kHz}$ and the quench frequency $f_{q}=8 \mathrm{MHz}$, has been considered. Fig. 14(a) shows the magnitude of the envelope of the input signal. Fig. 14(b) shows the magnitude of the envelope of the output signal. The results obtained through (33), using the LTV transfer function in (30), are overlapped with those obtained through a circuit-level envelope-transient simulation in commercial software. Fig. 14(c) compares the results of the LTV transfer function with standard time-domain integration and Fig 14(d) presents the same comparison based on an expanded view of a single pulse.

In a second test, a QPSK signal at the carrier frequency $f=2.7$ $\mathrm{GHz}$ has been considered. The symbol period agrees with the quench period and is given by $T_{s}=T_{q}=125 \mathrm{~ns}$. Fig. 15(a) shows the magnitude of the output signal, obtained through (33) and by means of circuit-level envelope-transient simulations, with overlapped results. Fig. 15(b) compares the phase of the input and output signals. In agreement with the derivations in [5], the SRO is able to follow the phase shifts.

Fig. 15(c) shows an expanded view of the amplitude and phase variations corresponding to two oscillation pulses. The $90^{\circ}$ phase increment in the input signal is properly followed by these two oscillation pulses. When the oscillation is off, there is a small output signal at $\omega$, due to the presence of the small input signal at the same frequency, with the input power $P_{i n}=-57$ $\mathrm{dBm}$. Then, the output signal exhibits a near-flat phase during the constant phase states of the QPSK modulation. However, during the oscillation grow up and decay there are significant phase changes, less pronounced when the pulse amplitude is about its maximum. The phase changes are due to the variation in the instantaneous-oscillation frequency. Since the fundamental frequency of the envelope-domain equations and LTV transfer function is kept fixed to $\omega$, this variation gives rise to a time-varying phase $\phi(t)$, which adds up to the phase changes induced by the QPSK-modulated input signal. The phase variations are strong in this transistor-based oscillator due the quench-signal modulation effects, since the resonance takes place between the inductor $L_{D}$ and the transistor output capacitance. The apparent phase discontinuity in the transistorbased oscillator is due to the wrap to $-180^{\circ}$ to $180^{\circ}$. Nevertheless, the phase increment induced by the QPSK signal is properly tracked by the SRO.

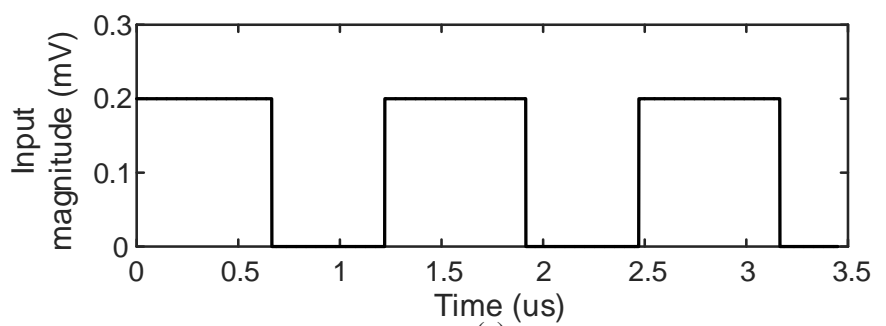

(a)

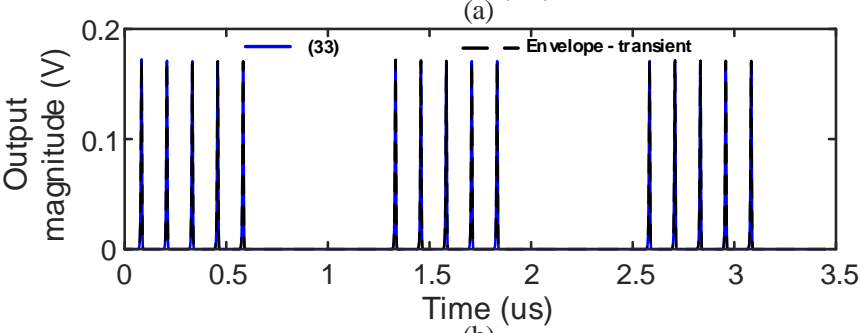

(b)

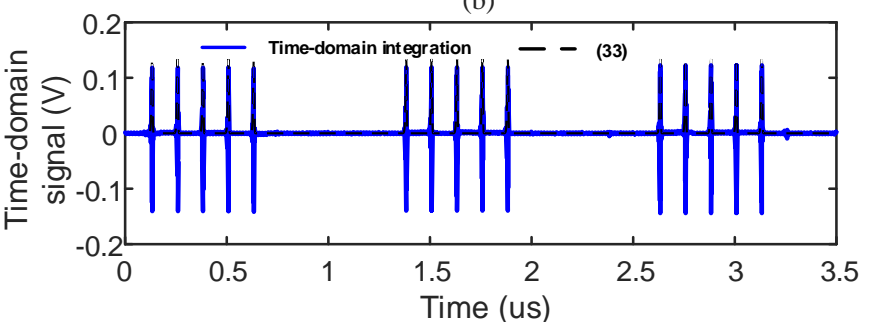

(c)

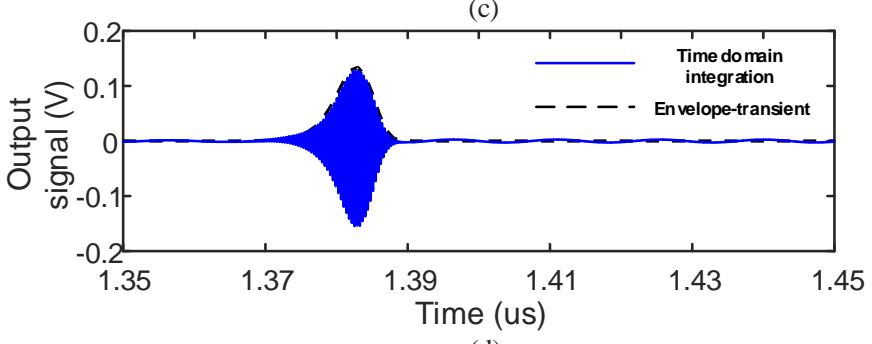

(d)

Fig. 14. Response to an on-off keying signal at the carrier frequency $f=2.7 \mathrm{GHz}$, at the modulation frequency $f_{\bmod }=800 \mathrm{kHz}$. The quench frequency is $f_{q}=8 \mathrm{MHz}$. (a) Magnitude of the envelope of the input signal. (b) Magnitude of the envelope of the output signal. Results obtained through (33), using the LTV transfer function in (30), are overlapped with those obtained through a circuit-level envelope-transient simulation in commercial software. (c) Comparison of the results of the LTV transfer function with standard timedomain integration. (d) Expanded view of a single pulse. 

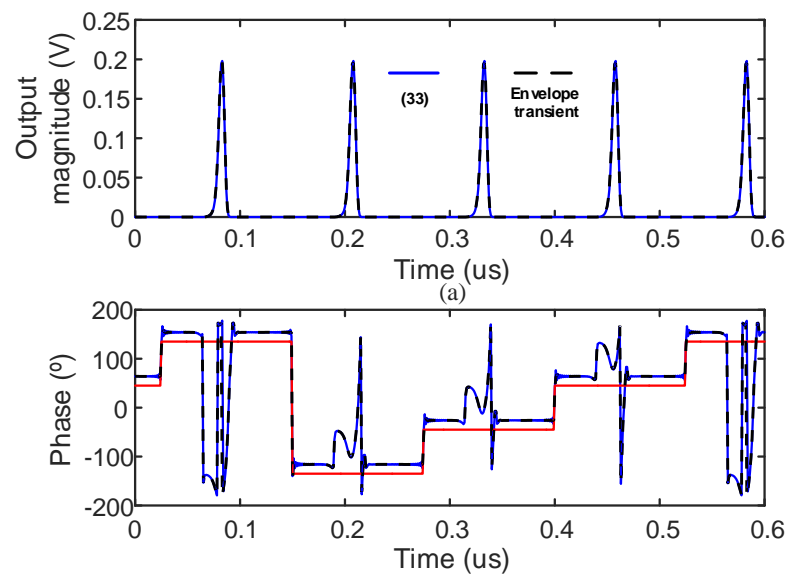

(b)

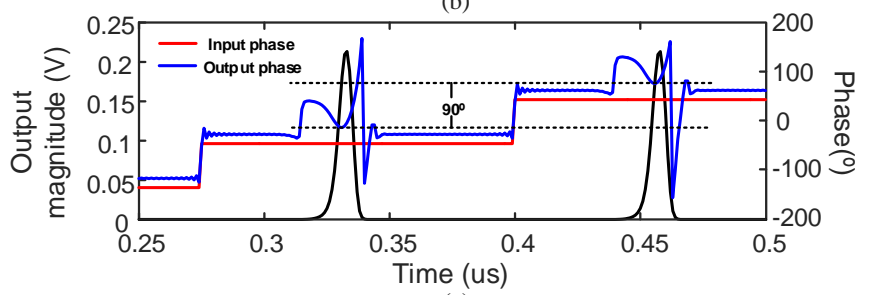

(c)

Fig. 15. QPSK signal at the carrier frequency $f=2.7 \mathrm{GHz}$. The symbol period agrees with the quench period and is given by $T_{s}=T_{q}=125 \mathrm{~ns}$. (a) Magnitude of the output signal, obtained through (33) and by means of circuit-level envelope-transient simulations, with overlapped results. (b) Comparison of the phase of the input and output signal. In the time intervals of interest, which are the ones with a pulse amplitude different from zero, the output phase predicted through (33) is overlapped with the one obtained through envelope-transient simulations. (c) Expanded view of the phase variations during two oscillation pulses. The $90^{\circ}$ phase increment in the input signal is properly followed by these two oscillation pulses.

TABLE III

ALGORITHM FOR THE CALCULATION OF THE SRO OUTPUT UNDER ARBITRARILY MODULATED INPUT SIGNALS

\begin{tabular}{|c|c|}
\hline Read Time_vector & $\begin{array}{l}\text { Reading of the vector of time } \\
\text { values exported from ADS }\end{array}$ \\
\hline $\begin{array}{l}T= \\
\max \text { (Time_vector) - } \\
\min (\text { Time_vector) }\end{array}$ & $\begin{array}{l}\text { Calculation of the duration of the } \\
\text { time interval }\end{array}$ \\
\hline $\begin{array}{l}N= \\
\text { length (Time_vector) }\end{array}$ & $\begin{array}{l}\text { Calculation of the number of } \\
\text { time points }\end{array}$ \\
\hline $\begin{array}{l}\text { Vin_ } t=A_{-} t^{*} \exp \text { (phase } \\
t \text { ) }\end{array}$ & $\begin{array}{l}\text { Definition of the complex- } \\
\text { envelope of the arbitrarily } \\
\text { modulated input signal }\end{array}$ \\
\hline $\begin{array}{l}\text { Read } \\
\text { Frequency_vector }\end{array}$ & $\begin{array}{l}\text { Reading of the frequency vector } \\
\text { exported from ADS. Prior to } \\
\text { exporting, this frequency vector } \\
\text { is translated doing } f=f_{\text {sweep }}-f_{p} \text {, } \\
\text { where } f_{\text {sweep }} \text { are the frequencies } \\
\text { in the sweep. }\end{array}$ \\
\hline $\begin{array}{l}\mathrm{fx}_{\mathrm{X}}= \\
\text { Frequency_vector }\end{array}$ & $\begin{array}{l}\text { Definition of the frequency } \\
\text { vector }\end{array}$ \\
\hline $\operatorname{Vin}{ }_{-} W=F F T\left(V i n_{-} t\right)$ & $\begin{array}{l}\text { Calculation of } V_{\text {in }}(\Omega) \text { through } \\
\text { FFT of } V_{\text {in }}(t)\end{array}$ \\
\hline for $n=1: N$ & $\begin{array}{l}\text { Beginning of the loop that } \\
\text { calculates } V_{\text {out }}\left(t_{n}\right)\end{array}$ \\
\hline
\end{tabular}

\begin{tabular}{|ll|}
\hline Read $\boldsymbol{f}, \boldsymbol{H}\left(\boldsymbol{t}_{\boldsymbol{n}}, \boldsymbol{f}\right)$ & Reading of the function \\
& $f, H\left[t_{n}, 2 \pi\left(f+f_{p}\right)\right]$ exported \\
& from ADS \\
Vout $(n)=$ & Calculation of the following IFT \\
IFF $\left(\mathrm{t} n, H .{ }^{*}\right.$ Vin_W) & integral: \\
& $V_{\text {out }}\left(t_{n}\right)=$ \\
& $\frac{1}{2 \pi} \int_{\omega_{\min }-\omega_{p}}^{\omega_{\max }-\omega_{p}} H\left(t_{n}, \Omega+\omega_{p}\right) V_{\text {in }}(\Omega) e^{j \Omega t_{n}} d \Omega$ \\
& \\
end &
\end{tabular}

\section{Measurements}

The transistor-based SRO has been experimentally characterized under an on-off keying modulation. The carrier frequency is $f=2.7 \mathrm{GHz}$, the modulation frequency is $f_{\text {mod }}=800 \mathrm{KHz}$ and the quench frequency is $f_{q}=8 \mathrm{MHz}$. The transistor is biased at the drain voltage $V_{d}=0.6 \mathrm{~V}$ and consumes the dc current $I_{d}=0.066 \mathrm{~A}$. The quench signal values are $V_{p}=455 \mathrm{mVpp}, V_{d c}=270 \mathrm{mVdc}$ and $f_{q}=8 \mathrm{MHz}$

The measurement set-up is shown in Fig. 15(a) and the photograph of the measured prototype is shown in Fig. 15(b). The RF input signal is generated using the ANRITSU MG3710A Vector Signal Generator, setting a square-pulse modulated signal with a carrier frequency of $f=2.7 \mathrm{GHz}$, a modulation frequency $f_{\text {mod }}=800 \mathrm{kHz}$ and an output power of $P_{R F}=-37 \mathrm{dBm}$. A directional coupler and an attenuator of 10 $\mathrm{dB}$ have been connected to the vector signal generator output to obtain the SRO input power $P_{i n}=-57 \mathrm{dBm}$. The transmitted port of the directional coupler has been directly connected to a DSO90804A Digital Storage Oscilloscope to observe the modulated input signal as well as the SRO output.

Fig. 17(a) presents the sinusoidal quench signal, which has been generated with the Agilent 81180B Arbitrary Waveform Generator. The RF signal, $R F_{\text {in }}(t)$, in Fig. $17($ b) was obtained by simply connecting the transmitted port of the directional coupler to the oscilloscope. Fig. 17(c) presents the attenuated RF signal spectrum, measured by directly connecting the output of the $10 \mathrm{~dB}$ attenuator to the Agilent E4407B Spectrum Analyzer. Note that prior to its introduction into the SRO, the RF signal passes through the coupled port of the directional coupler with $10 \mathrm{~dB}$ attenuation and an attenuator of $10 \mathrm{~dB}$.

The measured output signal is shown in Fig. 18(a). The "on" and "off" intervals are clearly distinguished by the magnitude of the output pulses. There are small-amplitude output pulses in the off intervals, due to noise. Fig. 18(b) compares the measured output amplitude with the one obtained through (33). Fig. 18(c) presents an expanded view of a single pulse. Measurement results are compared with those obtained through (33), and with circuit-level envelope-transient simulations. The disagreement with respect to the experimental results is considered relatively small and is attributed to inaccuracies in the models of the active and passive elements contained in the SRO circuit. 

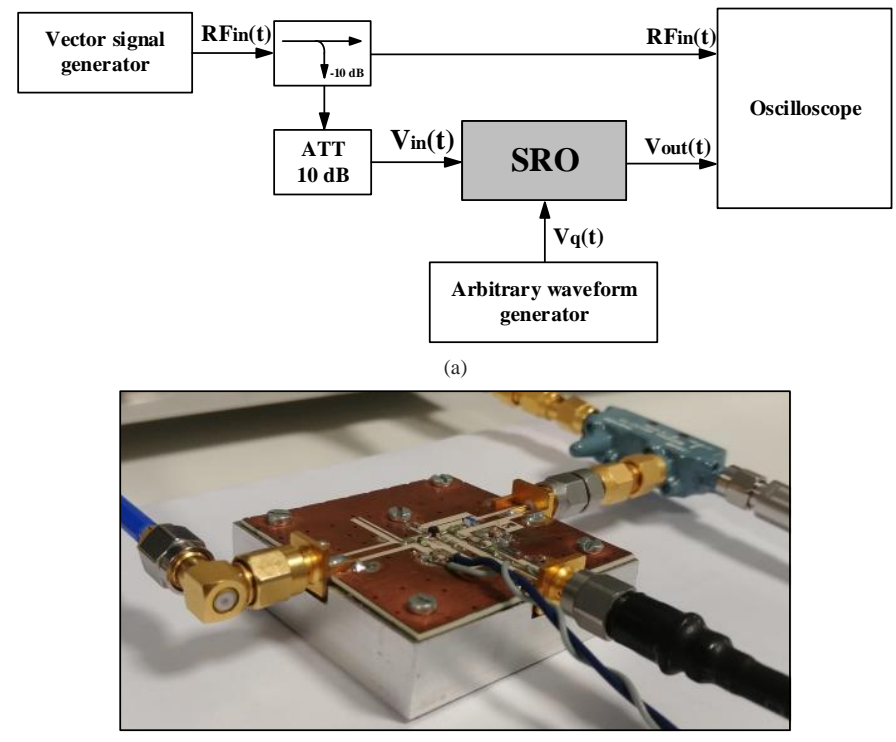

(b)

Fig. 16. (a) Measurements setup. The RF input signal is generated using the ANRITSU MG3710A Vector Signal Generator. A directional coupler and an attenuator of $10 \mathrm{~dB}$ have been connected to the generator output to obtain $P_{i n}=-57 \mathrm{dBm}$ at the SRO input. The transmission port of the directional coupler has been directly connected to a DSO90804A Digital Storage Oscilloscope to observe the modulated input signal as well as the SRO output. (b) Photograph of the measured prototype built on Rogers 4003C substrate (cr $=3.55, \mathrm{H}=32$ mils .
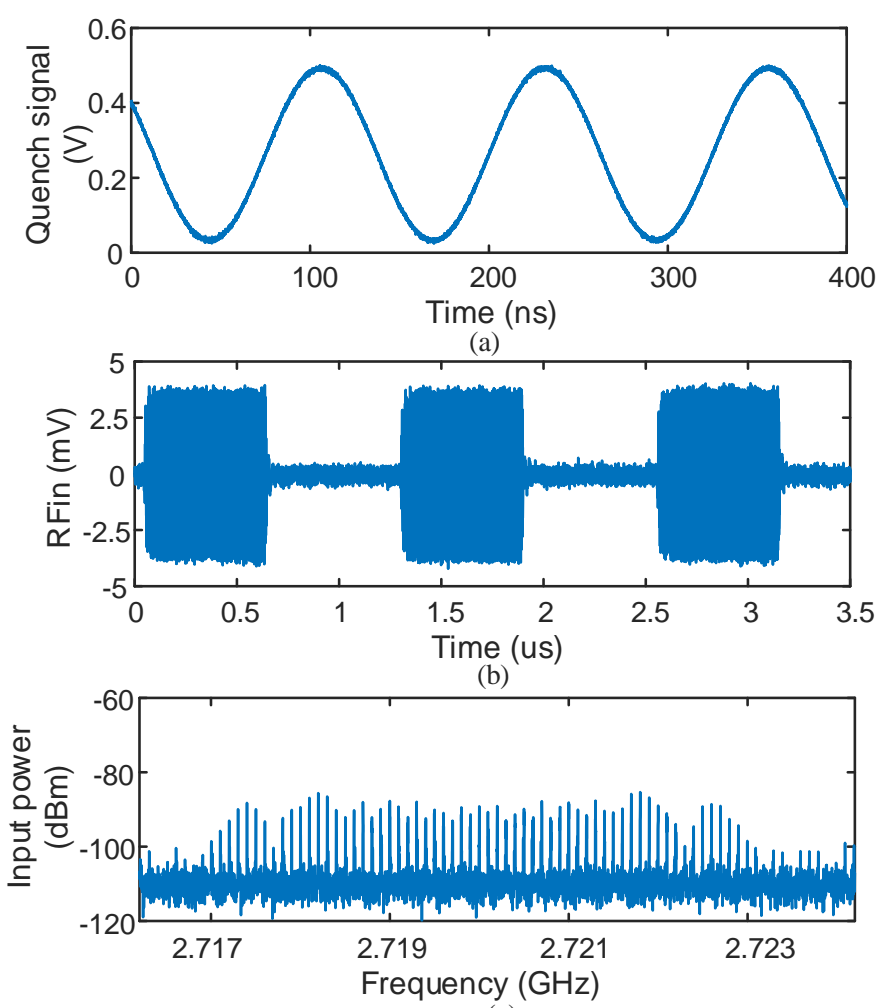

(c)

Fig. 17. Experimental characterization of the SRO, using the set-up in Fig. 16. (a) Sinusoidal quench signal with $V_{p}=455 \mathrm{mVpp}, V_{d c}=270 \mathrm{mVdc}$ and $f_{q}=$ $8 \mathrm{MHz}$, generated with the Agilent $81180 \mathrm{~B}$ Arbitrary Waveform Generator. (b) Input signal, $R F_{\text {in }}(t)$, extracted from the transmitted port of the directional coupler. (c) Input-signal spectrum, measured by directly connecting the output of the attenuator to the spectrum analyzer.
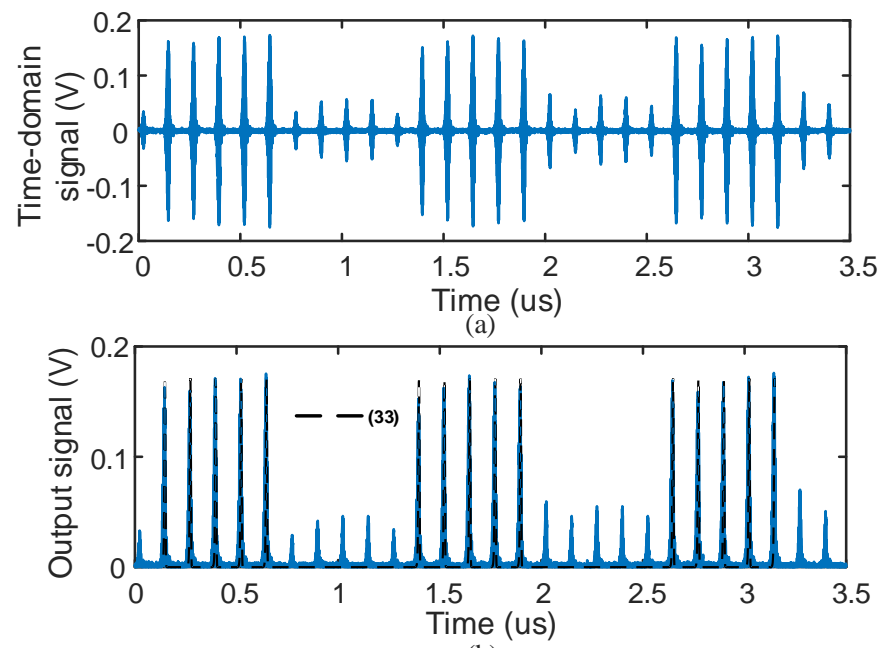

(b)

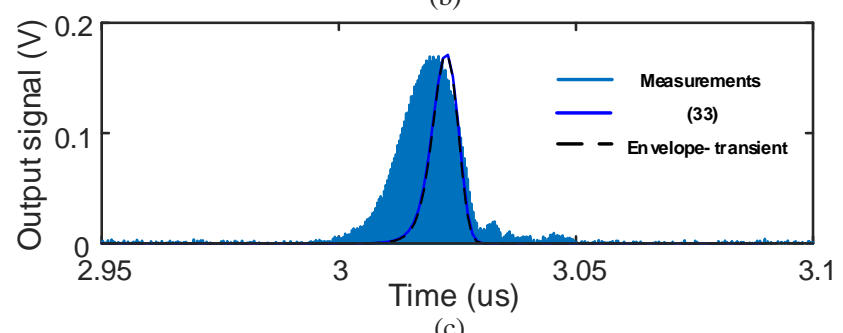

(c)

Fig. 18. Experimental output signal. (a) Time-domain measurement. (b) Comparison of the magnitude of the experimental output signal with the predictions by (33). (c) Expanded view of a single pulse. Comparison of the measurement results with those obtained through (33), and with circuit-level envelope-transient simulations.

\section{CONCLUSION}

An envelope-transient methodology for the analysis and modeling of super-regenerative oscillators has been presented. In a first stage, the stability of the non-oscillatory solution forced by the quench signal is analyzed through the calculation of its time-variant poles. This allows establishing the reference times of the SRO, specifically, the central time of the sensitivity interval and the time value at which the output envelope reaches its maximum magnitude. In a second stage, the full SRO response in linear regime is modeled with an LTV impulse response and its associated LTV transfer function. The calculation requires the frequency sweep of a small signal sinusoidal source in an envelope-transient simulation. The LTV impulse response enables a straightforward identification of the sensitivity interval and other essential characteristics of the SRO performance. The LTV transfer function enables the calculation of the SRO output under any small-signal input, with arbitrary carrier frequency and modulation. The results have been successfully validated with circuit-level envelopetransient simulations and with measurements.

\section{ACKNOWLEDGMENT}

Authors would like to acknowledge S. Sancho from University of Cantabria for helpful discussions. 


\section{REFERENCES}

[1] J. R. Whitehead, Super-regenerative receivers. Cambridge: University Press, 1950.

[2] F. X. Moncunill-Geniz, P. Pala-Schonwalder and O. Mas-Casals, "A generic approach to the theory of superregenerative reception," in IEEE Trans. Circuits Syst. I, Reg. Papers, vol. 52, no. 1, pp. 54-70, Jan. 2005.

[3] J. Bonet-Dalmau, F. X. Moncunill-Geniz, P. Pala-Schonwalder, F. del Aguila-Lopez and R. Giralt-Mas, "Frequency Domain Analysis of Superregenerative Receivers in the Linear and the Logarithmic Modes," in IEEE Trans. Circuits Syst. I, Reg. Papers, vol. 59, no. 5, pp. 1074-1084, May 2012.

[4] F. X. Moncunill-Geniz, P. Pala-Schonwalder, C. Dehollain, N. Joehl and M. Declercq, "An 11-Mb/s 2.1-mW Synchronous Superregenerative Receiver at $2.4 \mathrm{GHz}$, in IEEE Trans. Microw. Theory Techn., vol. 55, no. 6, pp. 1355-1362, June 2007.

[5] P. Palà-Schönwälder, J. Bonet-Dalmau, F. X. Moncunill-Geniz, F. del Águila-López and R. Giralt-Mas, "A Superregenerative QPSK Receiver," in IEEE Trans. Circuits Syst. I, Reg. Papers, vol. 61, no. 1, pp. 258-265, Jan. 2014.

[6] H. Ghaleb, P. V. Testa, S. Schumann, C. Carta and F. Ellinger, "A 160$\mathrm{GHz}$ Switched Injection-Locked Oscillator for Phase and Amplitude Regenerative Sampling," in IEEE Microw. Compon. Lett, vol. 27, no. 9, pp. 821-823, Sept. 2017.

[7] C. Carlowitz and M. Vossiek, "Demonstration of an efficient high speed communication link based on regenerative sampling," 2017 IEEE MTT-S International Microwave Symposium (IMS), Honololu, HI, 2017, pp. 7174.

[8] P. E. Thoppay, C. Dehollain and M. J. Declercq, "A 7.5mA $500 \mathrm{MHz}$ UWB receiver based on super-regenerative principle," ESSCIRC 2008 34th European Solid-State Circuits Conference, Edinburgh, 2008, pp. 382-385.

[9] V. D. Rezaei, S. J. Shellhammer, M. Elkholy and K. Entesari, "A fully integrated $320 \mathrm{pJ} / \mathrm{b}$ OOK super-regenerative receiver with $-87 \mathrm{dBm}$ sensitivity and self-calibration," 2016 IEEE Radio Frequency Integrated Circuits Symposium (RFIC), San Francisco, CA, 2016, pp. 222-225.

[10] M. Vossiek and P. Gulden, "The Switched Injection-Locked Oscillator: A Novel Versatile Concept for Wireless Transponder and Localization Systems," IEEE Trans. Microw. Theory Techn., vol. 56, no. 4, pp. 859866, Apr., 2008.

[11] M. Vossiek, T. Schafer and D. Becker, "Regenerative backscatter transponder using the switched injection-locked oscillator concept," 2008 IEEE MTT-S Int. Microwave Symp. Dig., Atlanta, GA, 2008, pp. 571-574.

[12] J. L. Bohorquez, A. P. Chandrakasan and J. L. Dawson, "FrequencyDomain Analysis of Super-Regenerative Amplifiers," in IEEE Trans. Microw. Theory Techn., vol. 57, no. 12, pp. 2882-2894, Dec. 2009.

[13] D. R. Frey, "Improved Super-Regenerative Receiver Theory", IEEE Trans. Circuits Syst. I, Reg. Papers, vol. 60, Issue: 12, Pages: 3267 3278, Dec. 2013.

[14] E. Ngoya and R. Larcheveque, "Envelop transient analysis: a new method for the transient and steady state analysis of microwave communication circuits and systems," IEEE MTT-S Int. Microwave Symp. Dig., San Francisco, CA, USA, 1996, vol.3, pp. 1365-1368.

[15] H. Brachtendorf, G. Welsch, R. Laur and A. BunseGerstner, "Numerical Steady-State Analysis of Electronic Circuits Driven By Multi-Tone Signals", Electrical Engineering: 79, pp.103-112, 1996.

[16] E. Ngoya, J. Rousset and D. Argollo, "Rigorous RF and microwave oscillator phase noise calculation by envelope transient technique," 2000 IEEE MTT-S Int. Microwave Symp. Dig., Boston, MA, USA, 2000, pp. 91 94 vol.1.

[17] K. S. Kundert, "Introduction to RF simulation and its application," in IEEE Journal of Solid-State Circuits, vol. 34, no. 9, pp. 1298-1319, Sep 1999.

[18] K. Kundert, J. White, A. Sangiovanni-Vincentelli, Steady-State Methods for Simulating Analog and Microwave Circuits, New York, NY, USA:Kluwer Academic Publishers, 1990.

[19] J. C. Pedro and N. B. Carvalho, "Simulation of RF circuits driven by modulated signals without bandwidth constraints," 2002 IEEE MTT-S Int. Microwave Symp. Dig., Seattle, WA, USA, 2002, pp. 2173-2176 vol.3.

[20] M. E. de Cos, A. Suarez and S. Sancho, "Envelope transient analysis of self-oscillating mixers," IEEE Trans. Microw. Theory Techn., vol. 52, no. 4, pp. 1090-1100, Apr., 2004.

[21] Tuhin Subhra Chakraborty; Satya Sudhakar Yedlapalli; Arun Naniyat; Young-Jun Hong; Sungjin Kim,"On the Modeling of Low Pass Characteristics of Super-Regenerative Receiver for High Speed
Simulation", IEEE Vehicular Technology Conference (VTC Fall), 2011, Pages: 1-6.

[22] Seyed Mohammadreza Fatemi; Mohammad Sharifkhani; Ali FotowatAhmady, "A Unified Solution for Super-Regenerative Systems With Application to Correlator-Based UWB Transceivers", IEEE Trans. Circuits Syst. I, Reg. Papers, Volume: 62, Issue: 4, Pages: 1033 - 1041, Apr. 2015

[23] L. A. Zadeh, "Frequency Analysis of Variable Networks," in Proceedings of the IRE, vol. 38, no. 3, pp. 291-299, March 1950.

[24] L. A. Zadeh, "Constant-Resistance Networks of the Linear VaryingParameter Type," in Proceedings of the IRE, vol. 39, no. 6, pp. 688-691, June 1951.

[25] L. A. Zadeh, "On stability of linear varying-parameter systems," Journal of Applied Physics, vol. 22, no. 4, pp. 402-405, 1951.

[26] T. S. Parker and L.O. Chua, Practical Numerical Algorithms for Chaotic Systems, Springer-Verlag, Berlin, 1989.

[27] K. S. Kundert and J. K. White, Computer aided analysis of Electronic circuits, Upper Saddle River, NJ: Prentice Hall, 1975.

[28] J. F. Oliveira and J. C. Pedro, "An Efficient Time-Domain Simulation Method for Multirate RF Nonlinear Circuits," IEEE Trans. Microw. Theory Techn., vol. 55, no. 11, pp. 2384-2392, Nov. 2007.

[29] J. Roychowdhury, "Analyzing circuits with widely separated time scales using numerical PDE methods," IEEE Trans. Circuits Syst. I, Fundam. Theory Appl. (1993-2003), vol. 48, no. 5, pp. 578-594, May 2001.

[30] L. Mandache, D. Topan, IG. Sirbu, "Universal Approach of the Modified Nodal Analysis for Nonlinear Lumped Circuits in Transient Behavior." Ao SI., Gelman L. (eds) Electrical Engineering and Applied Computing. Lecture Notes in Electrical Engineering, vol 90. Springer, Dordrecht.

[31] A. B. Carlson, Communication Systems, McGraw-Hill, New York, 1986.

[32] F. Ramirez, A. Suarez, I. Lizarraga and J. M. Collantes, "Stability Analysis of Nonlinear Circuits Driven With Modulated Signals," in IEEE Trans. Microw. Theory Techn., vol. 58, no. 4, pp. 929-940, April 2010.

[33] A. Suárez, Analysis and Design of Autonomous Microwave Circuits. Hoboken, NJ: Wiley IEEE Pres, 2009.

[34] J. Jugo, J. Portilla, A. Anakabe, A. Suárez, and J. M. Collantes, "Closedloop stability analysis of microwave amplifiers," IEEE Electron. Lett., vol. 37, no. 4, pp. 226-228, Mar., 2001.

[35] J. M. Collantes, I. Lizarraga, A. Anakabe, and J. Jugo, "Stability verification of microwave circuits through Floquet multiplier analysis," Proc. IEEE Asia-Pacific Conf. Circuits Syst., Tainan, Taiwan, 2004, pp. 997-100.

[36] N. Ayllon, J. M. Collantes, A. Anakabe, I. Lizarraga, S. Soubercaze-Pun, S. Forestier, "Systematic approach to the stabilization of multitransistor circuits", IEEE Trans. Microw. Theory Techn., vol. 59, no. 8, pp. 20732082, Aug. 2011.

[37] A. Suárez, S. Jeon and D. Rutledge, "Stability analysis and stabilization of power amplifiers," IEEE Microwave Magazine, vol. 7, pp. 51-65, Oct., 2006.

[38] A. Suárez and R. Melville, "Simulation-assisted design and analysis of varactor-based frequency multipliers and dividers," IEEE Trans. Microw. Theory Techn., vol. 54, pp. 1166-1179, Mar., 2006.

[39] J. Roychowdhury, "Reduced-order modelling of linear time-varying systems," 1998 IEEE/ACM International Conference on Computer-Aided Design. Digest of Technical Papers (IEEE Cat. No.98CB36287), San Jose, CA, USA, 1998, pp. 92-95.

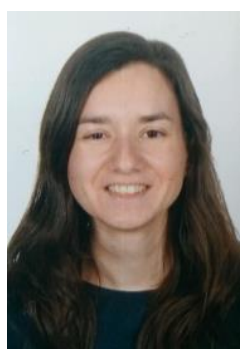

Silvia Hernández (S'17) was born in the Canary Islands, Spain. She received her M.S. degree in Telecommunication Engineering from the University of Las Palmas de Gran Canaria, Canary Islands, Spain, in 2015. The same year, she entered the Institute for the Technological Development and Innovation in Communications, University of Las Palmas de Gran Canaria, as a research assistant. In 2016, she joined the Communications Engineering Department, University of Cantabria, where she is currently working towards her Ph.D. degree.

Her research interests include stability, phase-noise analysis and the study of new methods for the analysis and design of nonlinear microwave circuits. 


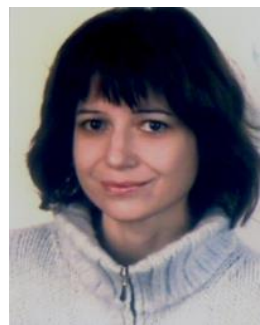

Almudena Suárez (M'96-SM'01-F'12) was born in Santander, Spain. She received the degree in electronic physics and the Ph.D. degree from the University of Cantabria, Santander, Spain, in 1987 and 1992, respectively, and the Ph.D. degree in electronics from the University of Limoges, France, in 1993. At present, she is a Full Professor at the University of Cantabria, and a member of its Communications Engineering Department. She has authored the book Analysis and design of autonomous microwave circuits for the publisher IEEE-Wiley and co-authored the book Stability analysis of microwave circuits for the publisher Artech-House. She belongs to the technical committees of the IEEE International Microwave Symposium and European Microwave Conference. She was an IEEE Distinguished Microwave Lecturer for the period 2006-2008. She is an associate editor of the IEEE Microwave Magazine. She is a member of the Board of Directors of EuMA and the Editor in Chief of International Journal of Microwave and Wireless Technologies from Cambridge University Press. 